\title{
Scaling of Hamiltonian walks on fractal lattices
}

\author{
Sunčica Elezović-Hadžic ${ }^{*}$ \\ University of Belgrade, Faculty of Physics, P.O. Box 368, Belgrade, Serbia \\ Dušanka Marčetiđđ \\ University of Banja Luka, Faculty of Science, Department of Physics, \\ M. Stojanovića 2, Banja Luka, Bosnia and Herzegovina \\ Slobodan Maletid $\ddagger$ \\ Institute of Nuclear Sciences Vinča, P.O.Box 522, Belgrade, Serbia
}

(Dated: September 13, 2018)

\begin{abstract}
We investigate asymptotical behavior of numbers of long Hamiltonian walks (HWs), i.e. selfavoiding random walks that visit every site of a lattice, on various fractal lattices. By applying an exact recursive technique we obtain scaling forms for open HWs on 3-simplex lattice, Sierpinski gasket, and their generalizations: Given-Mandelbrot (GM), modified Sierpinski gasket (MSG) and $n$-simplex fractal families. For GM, MSG and $n$-simplex lattices with odd values of $n$, the number of open HWs $Z_{N}$, for the lattice with $N \gg 1$ sites, varies as $\omega^{N} N^{\gamma}$. We explicitly calculate the exponent $\gamma$ for several members of GM and MSG families, as well as for $n$-simplices with $n=3,5$, and 7. For $n$-simplex fractals with even $n$ we find different scaling form: $Z_{N} \sim \omega^{N} \mu^{N^{1 / d_{f}}}$, where $d_{f}$ is the fractal dimension of the lattice, which also differs from the formula expected for homogeneous lattices. We discuss possible implications of our results on studies of real compact polymers.
\end{abstract}

PACS numbers: 82.35.Lr, 61.25.Hq, 05.45.Df

\section{INTRODUCTION}

Self-avoiding walks (SAWs) have long been used in the studies of configurational statistics of polymer chains in solution [1]. Due to excluded volume effect, at high temperatures $T$ (good solvent) long polymer chains are in swollen configurations. At low temperatures (poor solvent) polymers are in a collapsed state, caused by the attractive interactions of different sections of a polymer, mediated by a solvent. The transition between these two states occurs at the so-called $\theta$ temperature, at which excluded volume and attractive forces balance. Whereas the swollen and $\theta$ phases has been well investigated by now, the entropic scaling of the collapsed phase is still an open issue. A closely related problem is the scaling of Hamiltonian walks (HWs), which are SAWs that visit all the sites of the underlying lattice. HWs are believed to represent the $T=0$ limit of collapsed polymers, and they are also used in the studies of polymer melting [2], as well as in the context of protein folding [3] .

The number $Z_{N}$ of HWs on homogeneous lattices with $N \gg 1$ sites is expected to behave as

$$
Z_{N} \sim \omega^{N} \mu_{S}^{N^{\sigma}} N^{a}
$$

Here $\sigma=(d-1) / d$, where $d$ is the dimensionality of the lattice, $\mu_{S}$ is some constant less than 1 , and $\omega$ is the

\footnotetext{
*Electronic address: suki@ff.bg.ac.yu

${ }^{\dagger}$ Electronic address: dusamar@netscape.net

${ }^{\ddagger}$ Electronic address: supersloba@vin.bg.ac.yu
}

connectivity constant, defined as

$$
\ln \omega=\lim _{N \rightarrow \infty} \frac{\ln Z_{N}}{N} .
$$

Proposed scaling form for HWs differs from the ordinary SAW case (swollen polymer), where average number of $N$-step SAWs, for large $N$, behaves as $\omega^{N} N^{a}$, and where the critical exponent $a$ depends only on $d$ (which is not the case for $\mathrm{HWs}$ ). The term $\mu_{S}^{N^{\sigma}}$ in the $\mathrm{HW}$ case is expected on the basis of the exact study of HWs on the Manhattan lattice [4], as well as on the conjecture that collapsed polymer (globule) has a sharp boundary, so that a surface tension term should arise 5]. The scaling form (1.1) was confirmed by Owczarek [6] for collapsed partially directed SAWs on the square lattice. Baiesi et al 7] recently performed extensive Monte Carlo simulations, which gave strong evidence that (1.1) is also satisfied for undirected collapsed SAWs on the square lattice. There are hardly any results for higher dimensional lattices. To the best of our knowledge, the only clear indication that for collapsed SAWs on three-dimensional lattices there exists a surface term, as predicted by (1.1), was obtained by Grassberger and Hegger 8] via Monte Carlo simulations. There are also no results concerning scaling form for the collapsed SAWs on disordered lattices. Having all that in mind it might be useful to study HWs on fractals.

Fractal lattices are somehow intermediate between homogeneous and disordered ones, and their hierarchical and scale invariant structure often allows an exact recursive treatment of various physical phenomena. The ordinary SAW model (corresponding to the polymer chain at high temperatures) has been studied extensively in 
the past on different fractals [9]-20]. These studies contribute to a better understanding of how dimension and topological structure of underlying space affect the critical behavior of SAWs in general. There are fewer papers about self-interacting SAWs on fractals 21]-[25], and neither of them establishes the explicit form of the partition function at low temperatures. The closely related problem of finding the number of walks in the limiting HW case was analyzed only for closed walks on some fractals [26, 27]. Here for the first time we investigate the scaling forms for the number of open HWs on a set of fractal lattices.

The paper is organized as follows. In Sec. II we describe the technique used for an exact enumeration of closed HWs on the cases of 3-simplex lattice and Sierpinski gasket fractal, and extend it for enumeration of open HWs on these lattices. In Sections [II and IV this technique is generalized for enumeration of HWs on GivenMandelbrot (GM) and modified Sierpinski gasket (MSG) families of fractals. We find that there is no surface term in the scaling form for the number of HWs on neither of these lattices. For the number of closed HWs on MSG fractals we show that it varies as $\omega^{N}$, which is the same as in the case of GM fractals [27]. For open HWs we obtain scaling form $\omega^{N} N^{\gamma}$ for both fractal families, with $\omega$ and $\gamma$ depending on the particular parameters of the fractals. Furthermore, exponent $\gamma$ does not have the same value for corresponding fractals from these two families, as is the case for ordinary SAWs [10, 28]. Similar violation of the universality was found for two-dimensional (2d) homogeneous lattices [29]. We extend our analysis to higher dimensional $n$-simplex lattices in Sec. $\mathrm{V}$, where general exact scheme for obtaining numbers of HWs is described. It turns out that scaling form for HWs strongly depends on the parity of the fractal parameter $n$. In particular, for lattices with odd values of $n$ we find the same scaling form as for HWs on GM and MSG fractals, whereas for even $n$ we get $Z_{N} \sim \omega^{N} \mu^{N^{1 / d_{f}}}$, with $d_{f}$ being the fractal dimension of the lattice, and different values of $\mu$ for closed and open HWs. All obtained results are summarized in Sec. VI, and some technical details are given in the appendixes.

\section{HAMILTONIAN WALKS ON 3-SIMPLEX LATTICE AND SIERPINSKI GASKET FRACTAL}

In order to explain the method used in this paper for obtaining the scaling forms of numbers of both closed and open HWs we start with the simplest case: HWs on the 3-simplex lattice, and proceed with HWs on the Sierpinski gasket.

\section{A. 3-simplex lattice}

To obtain the 3-simplex lattice [30] one starts with a complete graph of three points and replaces each of these

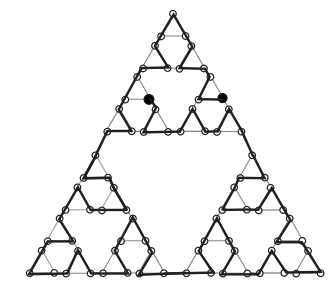

(a)

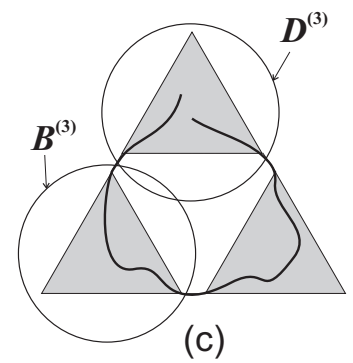

(c)

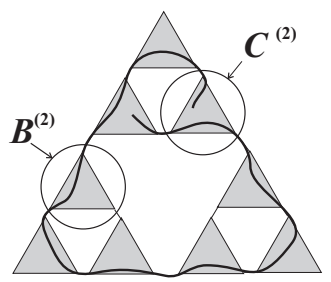

(b)

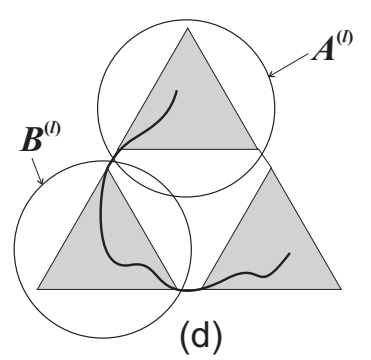

FIG. 1: (a) An open HW on a 3-simplex of order $l=4$. The first (b) and the second step (c) of the coarse-graining process. Gray triangles in (b) and (c) represent 3-simplices of order $l=2$ and $l=3$, respectively, whereas curved lines correspond to the coarse-grained walk. Different types of configurations within the third and the second order simplices are encircled. (d) Open HW on 3-simplex of order $(l+1)$, consisting of two $A$-type parts and one $B$-type part within the corresponding simplices of order $l$ (gray triangles).

points by a new complete graph of three points. The subsequent stages are constructed self-similarly, by repeating this procedure. After $l$ such iterations one obtains 3-simplex of order $l$ (see Fig. 1(a)), whereas the complete 3 -simplex lattice is obtained in the limit $l \rightarrow \infty$. The number of vertices within the $l$ th order 3 -simplex is $N_{l}=3^{l}$, and the fractal dimension of the lattice is $d_{f}=\ln 3 / \ln 2$.

In Fig. 1(a) we give an example of open HW on a 3simplex of order $l=4$. Performing a coarse-graining process one notices in Fig. 1(b) that this walk can be decomposed into parts corresponding to the second order simplices, which have two possible configurations: one traversing the simplex ( $B$-type configuration) and the other consisting of two strands, one of which is traversing the simplex, and the other with one end at a corner vertex of the same simplex, and the second end anywhere within it ( $C$-type configuration). The coarse-graining can be applied once more (Fig. 1(c)), leading to a HW consisting of three parts corresponding to three simplices of order $l=3$, two of them being of the type $B$, and the third consisting of two strands ("legs"), each of them having one end-point at the corner vertex of the corresponding simplex and the other within it ( $D$-type configuration). In a similar way, it is not difficult to see that each open HW on the $(l+1)$ th order simplex can be decomposed in two ways, either as in the sample shown in Fig. 1(c), or as in Fig. 1(d), with one $B$-type part and two $A$-type 


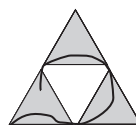

$C^{(1)}\left(B^{(1)}\right)^{2}$
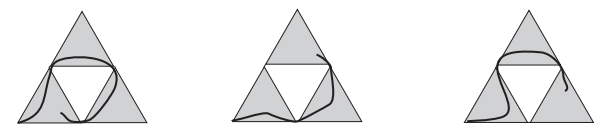

$A^{(l)}\left(B^{(l)}\right)^{2}$

(a)

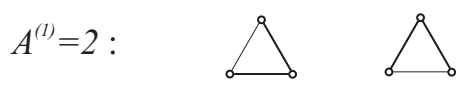

(b)

FIG. 2: (a) Four possible configurations of A-type HWs on a 3-simplex of order $(l+1)$, with the corresponding numbers of HWs. Gray triangles represent the $l$ th order simplex. (b) Two possible A-type HWs on the first order 3-simplex. Small circles represent sites the lattice consists of.

parts (one strand with one end at the corner vertex and the other end anywhere within the corresponding $l$ th order simplex). If we denote the number of open $\mathrm{HWs}$ on the $(l+1)$ th order simplex by $Z_{O}^{(l+1)}$, then the following relation is valid:

$$
Z_{O}^{(l+1)}=3 B^{(l)}\left[\left(A^{(l)}\right)^{2}+B^{(l)} D^{(l)}\right],
$$

where $A^{(l)}, B^{(l)}$ and $D^{(l)}$ are the numbers of HWs of types $A, B$ and $D$ within the $l$-th order simplex, respectively. Decomposition of closed HWs is even simpler, leading to the recursion relation:

$$
Z_{C}^{(l+1)}=\left(B^{(l)}\right)^{3}
$$

where $Z_{C}^{(l+1)}$ is the overall number of the closed HWs on the simplex of order $(l+1)$. Number $C^{(l)}$ of $C$-type HWs does not contribute directly to $Z_{O}^{(l+1)}$ and $Z_{C}^{(l+1)}$, but one can obtain (see Fig. 2) the following recursion relations:

$$
\begin{aligned}
& A^{(l+1)}=2 B^{2}(A+C), \\
& B^{(l+1)}=B^{3}, \\
& C^{(l+1)}=B^{2}(A+3 C), \\
& D^{(l+1)}=B\left(4 A C+2 A^{2}+3 B D+6 C^{2}\right),
\end{aligned}
$$

for the numbers of HWs corresponding to simplices of two successive orders (where we have omitted index $(l)$ on the right-hand side of the relations). Iterating these relations, starting with the initial values $A^{(1)}=2, B^{(1)}=1$, $C^{(1)}=1$, and $D^{(1)}=2$, one can calculate $A^{(l)}, B^{(l)}$, $C^{(l)}$, and $D^{(l)}$ for any $l$, and, consequently, the numbers $Z_{O}^{(l+1)}$ and $Z_{C}^{(l+1)}$. Furthermore, these relations can be exactly analyzed, as follows. From (2.4), and the corresponding initial condition, trivially follows $B^{(l)} \equiv 1$, which also transforms the set of relations (2.3) i (2.5) for one-leg walks $A$ and $C$ into a simple system of difference equations:

$$
A^{(l+1)}=2\left(A^{(l)}+C^{(l)}\right), \quad C^{(l+1)}=A^{(l)}+3 C^{(l)},
$$

whose solution is

$$
A^{(l)}=\frac{1}{3}\left(4^{l}+2\right), \quad C^{(l)}=\frac{1}{3}\left(4^{l}-1\right) .
$$

Then, from recursion relation (2.6) straightforwardly follows the difference equation for the number of two-leg walks $D$ :

$$
D^{(l+1)}=\frac{2}{3} 4^{2 r}+3 D^{(l)}
$$

whose solution is

$$
D^{(l)}=\frac{4}{39} 16^{r}+\frac{3}{13} 3^{r}-\frac{1}{3} .
$$

Finally, putting (2.7), (2.8), and $B^{(l)}=1$ in formulas for the overall numbers of open (2.1) and closed (2.2) HWs one gets

$$
Z_{O}^{(l)}=\frac{25}{16 \cdot 39} 16^{l}+\frac{1}{3} 4^{l}+\frac{3}{13} 3^{l}+\frac{1}{3}, \quad Z_{C}^{(l)}=1 .
$$

From the last equation trivially follows that connectivity constant is $\omega=1$ and $Z_{C}^{(l)}=\omega^{N_{l}}$ for every $l$, whereas the number of open HWs for $l \gg 1$ behaves according to the asymptotic formula

$$
Z_{O}^{(l)} \sim N_{l}^{\frac{\ln 16}{\ln 3}}=\omega^{N_{l}} N_{l}^{\gamma}
$$

where $N_{l}=3^{l}$ is the number of vertices of the 3 -simplex of order $l$, and $\gamma=\ln 16 / \ln 3=2.52372 \ldots \ldots$

\section{B. Sierpinski gasket}

In a similar way, one can analyze HWs on the Sierpinski gasket (SG) lattice. SG is a well known fractal lattice, which can be constructed recursively, starting with the generator (gasket of order $r=1$ ), which consists of three unit equilateral triangles, arranged to form a twice larger triangle (see Fig. 3(a)). The subsequent fractal stages are constructed self-similarly, by replacing each of the unit triangles of the initial generator with a new generator. To obtain the $r$ th-stage fractal lattice $(r$ th order gasket), this process of construction has to be repeated $(r-1)$ times, and the complete fractal is obtained in the limit $r \rightarrow \infty$. The numbers of sites on the $r$ th order gasket is equal to $N_{r}=\frac{3}{2}\left(3^{r}+1\right)$. SG resembles 3 -simplex lattice and indeed has the same fractal dimension $d_{f}=\ln 3 / \ln 2$. An open HW on a third order gasket is shown in Fig. 3, together with its coarse-grained versions. Comparing with Fig. 1] one can observe that larger number of types of possible HW configurations exists on SG than in the case of 3 -simplex lattice. There 


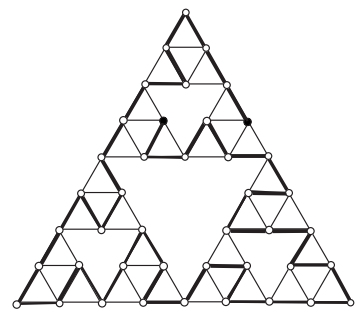

(a) (b)
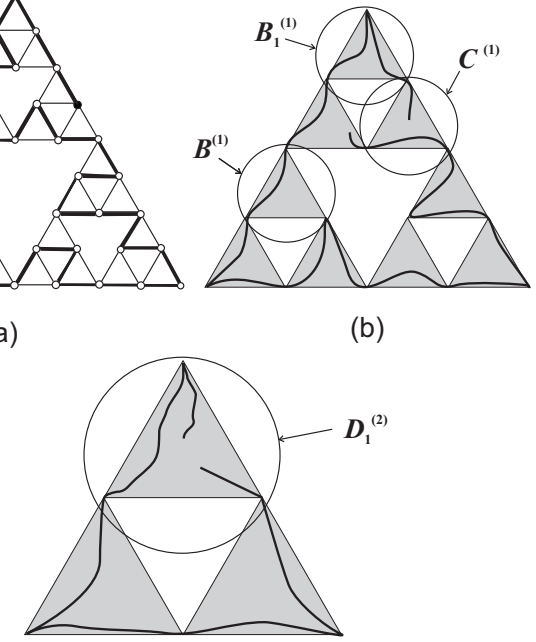

(c)

FIG. 3: (a) An open Hamiltonian walk on Sierpinski gasket of order $r=3$. Vertices corresponding to the end-points of the walk are colored black, for the sake of easier recognition. The first (b) and the second step (c) of coarse-graining process. Gray triangles in (b) and (c) represent gaskets of order $r=1$ and $r=2$, respectively, whereas curved heavy lines correspond to the coarse-grained walk. Different kinds of configurations within the first and the second order gaskets are encircled.

are exactly 8 different types of walks, and they are depicted in Fig. 4 The corresponding numbers of HWs on the $r$ th-order gasket will be denoted by $A^{(r)}, A_{1}^{(r)}, A_{2}^{(r)}$, $B^{(r)}, B_{1}^{(r)}, C^{(r)}, D^{(r)}$, and $D_{1}^{(r)}$. These numbers fulfill the following recursion relations:

$$
\begin{aligned}
A^{(r+1)} & =2\left(B^{2} C+A B B_{1}+A_{1} B^{2}\right), \\
A_{1}^{(r+1)} & =A B_{1}^{2}+2 A_{1} B B_{1}+2 A_{2} B^{2}+2 B B_{1} C,(2.11) \\
A_{2}^{(r+1)} & =A_{1} B_{1}^{2}+2 A_{2} B B_{1}+B_{1}{ }^{2} C, \\
B^{(r+1)} & =2 B^{2} B_{1}, \quad B_{1}^{(r+1)}=2 B B_{1}^{2}, \\
C^{(r+1)} & =A B_{1}^{2}+2 B\left(A_{1} B_{1}+A_{2} B+3 B_{1} C\right), \\
D^{(r+1)} & =4 A B_{1} C+4 A B A_{2}+4 A_{1} B C+4 B B_{1} D \\
& +4 A B_{1} A_{1}+2 B\left(3 C^{2}+2 B D_{1}+A_{1}^{2}\right), \\
D_{1}^{(r+1)} & =4 A_{1} A_{2} B+2 A A_{2} B_{1}+2 A_{1} C B_{1}+4 B B_{1} D_{1} \\
& +4 A_{2} C B+3 C^{2} B_{1}+A_{1}^{2} B_{1}+B_{1}^{2} D,
\end{aligned}
$$

with the initial values: $A^{(1)}=4, A_{1}^{(1)}=6, A_{2}^{(1)}=3$, $B^{(1)}=3, B_{1}^{(1)}=2, C^{(1)}=4, D^{(1)}=6$, and $D_{1}^{(1)}=5$. Iterating recursion relations (2.10)-(2.16) one can calculate numbers of all possible types of HWs, in principle for any $r$, and then eventually find the overall number $Z_{O}^{(r+1)}$ of open HWs on the $(r+1)$ th order gasket, by putting them into the formula:

$$
Z_{O}^{(r+1)}=12 A_{1}^{(r)} A_{2}^{(r)} B_{1}^{(r)}+6\left(B_{1}^{(r)}\right)^{2} D_{1}^{(r)} .
$$

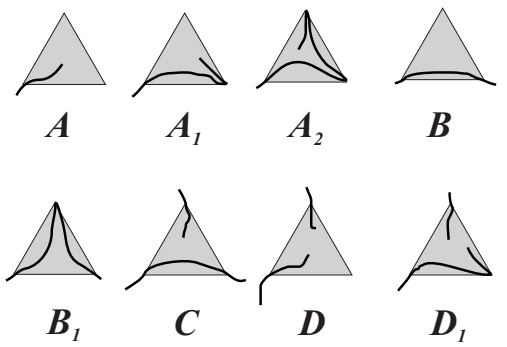

FIG. 4: Possible types of HWs on $r$ th order gaskets (gray triangles), needed to decompose any $\mathrm{HW}$ on gasket of order $r+1$.

However, this is a tedious task, since all of these numbers quickly become extremely large. To avoid that, we analyze recursion relations in a similar manner as was done for 3-simplex lattice.

Introducing new variables: $x_{r}=A^{(r)} / B^{(r)}, x_{1 r}=$ $A_{1}^{(r)} / B^{(r)}, x_{2 r}=A_{2}^{(r)} / B^{(r)}, y_{r}=C^{(r)} / B^{(r)}, z_{r}=$ $D^{(r)} / B^{(r)}, \quad z_{1 r}=D_{1}^{(r)} / B^{(r)}$, and noticing that $B_{1}^{(r)} / B^{(r)}=2 / 3$ is exactly satisfied for any $r$, from (2.10)-(2.16) one gets the new recursion relations:

$$
\begin{gathered}
\left(\begin{array}{c}
x^{\prime} \\
x_{1}^{\prime} \\
x_{2}^{\prime} \\
y^{\prime}
\end{array}\right)=\left(\begin{array}{cccc}
1 & \frac{3}{2} & 0 & \frac{3}{2} \\
\frac{1}{3} & 1 & \frac{3}{2} & 1 \\
0 & \frac{1}{3} & 1 & \frac{1}{3} \\
\frac{1}{3} & 1 & \frac{3}{2} & 3
\end{array}\right)\left(\begin{array}{c}
x \\
x_{1} \\
x_{2} \\
y
\end{array}\right), \\
\left(\begin{array}{c}
z^{\prime} \\
z_{1}^{\prime}
\end{array}\right)=\left(\begin{array}{cc}
2 & 3 \\
\frac{1}{3} & 2
\end{array}\right)\left(\begin{array}{c}
z \\
z_{1}
\end{array}\right)+\left(\begin{array}{c}
f_{1} \\
f_{2}
\end{array}\right),
\end{gathered}
$$

where

$$
\begin{aligned}
& f_{1}=2 x x_{1}+\frac{3}{2} x_{1}^{2}+3 x x_{2}+2 x y+3 x_{1} y+\frac{9}{2} y^{2}, \\
& f_{2}=\frac{1}{2} x_{1}^{2}+x x_{2}+3 x_{1} x_{2}+x_{1} y+3 x_{2} y+\frac{3}{2} y^{2} .
\end{aligned}
$$

From (2.18) for $r \gg 1$ follows $x_{r}, x_{1 r}, x_{2 r}, y_{r} \sim \lambda^{r}$, where $\lambda=4$ is the only eigenvalue of the $4 \times 4$ matrix of this recursion relation which is larger than 1 , and then, from (2.19), we find $z_{r}, z_{1 r} \sim 4^{2 r}$.

To obtain the asymptotic formula for the number of open HWs, we express $Z_{O}^{(r+1)}$ from (2.17) as

$$
Z_{O}^{(r+1)}=\frac{8}{3}\left(B^{(r)}\right)^{3}\left(3 x_{1 r} x_{2 r}+z_{1 r}\right) .
$$

Since in [27] it was exactly shown that $B^{(r)}=\operatorname{const} \omega^{N_{r}}$, with $\omega=12^{1 / 9}$, one finally gets the scaling form: $Z_{O}^{(r)} \sim$ $\omega^{N_{r}} N_{r}^{\gamma}$, with $\gamma=\ln 16 / \ln 3=2.52372 \ldots$ For the sake of comparison, we have generated $Z_{O}^{(r)}$ by direct numerical iteration, for $r$ up to 100. In Fig. 5 we depict $\left(\ln Z_{O}^{(r)}\right) / N_{r}$, as a function of $\left(\ln N_{r}\right) / N_{r}$, for $21 \leq r \leq 30$. As one can see, value of $\left(\ln Z_{O}^{(r)}\right) / N_{r}$ for this range of $r$ is quite close to $\ln \omega$, whereas by fitting these data to linear function one obtains approximate value: $\gamma_{f i t}=2.3$. For 


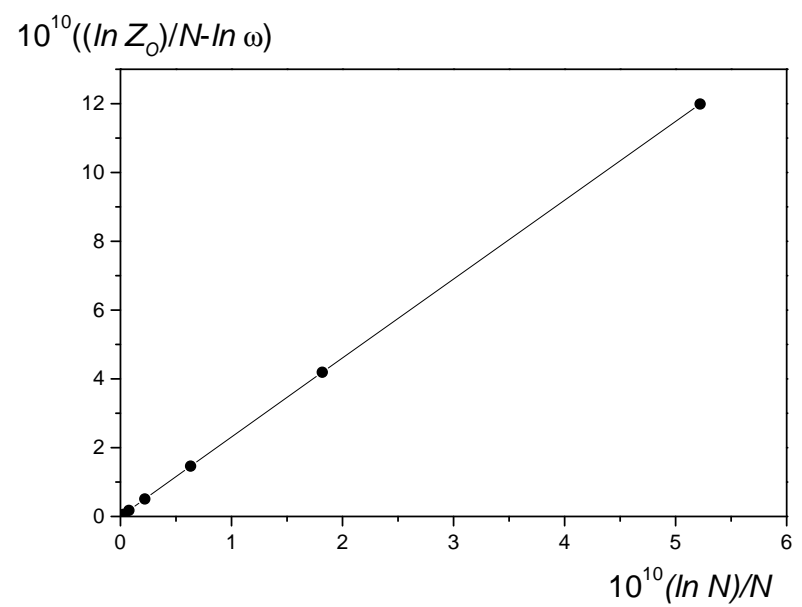

FIG. 5: Values of $\left(\ln Z_{O}^{(r)}\right) / N_{r}$, found by direct numerical iteration of relations (2.10)-(2.16) and (2.17), for $21 \leq r \leq 30$. Line connecting the points has been obtained by linear fitting, and $\omega=12^{1 / 9}$.

larger number of iterations $\gamma_{f i t}$ becomes closer to the exact value of $\gamma$. For instance, fitting the numerical data for $91 \leq r \leq 100$ gives $\left(\gamma-\gamma_{f i t}\right) / \gamma=2 \%$.

Scaling form obtained for the number of open HWs on $\mathrm{SG}$ is the same as the one found for the 3-simplex lattice. As was shown in [27], the numbers of closed HWs on SG scale as $\omega^{N_{r}}$, again the same as in the case of 3simplex. Equality of the exponents for SG and 3-simplex is in accord with the fact that SAWs on these two lattices belong to the same universality class [28]. On the other hand, it is known that exponents for HWs on different $2 \mathrm{~d}$ Euclidean lattices have different values 29], which is explained to be a consequence of the frustration, induced by the strong constraint that all the sites must be visited. It is believed that a relevant physical measure of this frustration is the number of contacts per monomer, i.e. vertex pairs which are not adjacent along the HW, but are the nearest neighbors on the lattice. Nevertheless, the number of contacts on 3-simplex is one, whereas it is two on SG, so that one could have expected different values of $\gamma$. In order to gain a deeper insight into the problem of universality and frustration of HWs on lattices embedded in $2 \mathrm{~d}$ space, in the next two sections we analyze the asymptotic behavior of HWs on the appropriate generalizations of 3-simplex and SG fractals.

\section{HAMILTONIAN WALKS ON GIVEN-MANDELBROT FRACTALS}

One possible way to generalize the SG fractal is to start with a generator that consists of $b(b+1) / 2$ unit equilateral triangles, arranged to form a $b$ times larger triangle. Enlarging the generator $b$ times and substitut- ing the smallest triangles with the generator, and then repeating this procedure recursively ad infinitum, one obtains fractal lattice characterized with the integer $b$. For $b=2,3, \ldots, \infty$ the complete so called Given-Mandelbrot (GM) family of fractals is obtained [31]. SG is the first member of this family, with the scaling parameter $b=2$. The fractal dimension of GM fractal with scaling parameter $b$ is equal to $d_{f}=\ln [b(b+1) / 2] / \ln b$, and the number of sites $N_{r}$ at the $r$ th stage of fractal construction is

$$
N_{r}=\frac{b+4}{b+2}\left[\frac{b(b+1)}{2}\right]^{r}+2 \frac{b+1}{b+2} .
$$

The overall number of open HWs on the $(r+1)$ th stage of construction of any GM fractal can be expressed in terms of numbers of $8 \mathrm{HW}$ types within the $r$ th order stage, in a similar manner as in the case of SG (Fig. 4). Recursion relations for numbers of $B$, and $B_{1}$-type walks on two successive stages of fractal construction have the following form:

$$
B^{\prime}=p B^{\frac{b(b-1)}{2}+1} B_{1}{ }^{b-1}, \quad B_{1}^{\prime}=p B^{\frac{b(b-1)}{2}} B_{1}{ }^{b},
$$

as was shown in [27], whereas the numbers of one-leg configurations: $A, A_{1}, A_{2}$, and $C$, satisfy a closed set of recursion relations, which can be put in matrix form as:

$$
\left(\begin{array}{l}
A^{\prime} \\
A_{1}^{\prime} \\
A_{2}^{\prime} \\
C^{\prime}
\end{array}\right)=\left(\begin{array}{llll}
a_{11} & a_{12} & a_{13} & a_{14} \\
a_{21} & a_{22} & a_{23} & a_{24} \\
a_{31} & a_{32} & a_{33} & a_{34} \\
a_{41} & a_{42} & a_{43} & a_{44}
\end{array}\right)\left(\begin{array}{c}
A \\
A_{1} \\
A_{2} \\
C
\end{array}\right),
$$

where $a_{i j}$ are polynomials in $B^{(r)}$ and $B_{1}^{(r)}$. In Appendix $\mathrm{A}$ we prove that each of these polynomials has only one term, which is of the form $K_{i j}\left[B^{(r)}\right]^{\beta}\left[B_{1}^{(r)}\right]^{\delta}$, where $\beta+\delta=b(b+1) / 2-1$. The coefficients $K_{i j}$ depend only on $b$, and each of them can be expressed in terms of the number $p$ (appearing in equations (3.2)), and additional 8 numbers: $a, a_{1}, a_{2}, c, a^{\prime}, a_{1}^{\prime}, a_{2}^{\prime}, c^{\prime}$. Introducing new variables: $x_{r}=A^{(r)} / B^{(r)}, x_{1 r}=A_{1}^{(r)} / B^{(r)}$, $x_{2 r}=A_{2}^{(r)} / B^{(r)}, y_{r}=C^{(r)} / B^{(r)}$, relation (3.3) gives the new recursive relation:

$$
\left(\begin{array}{c}
x^{\prime} \\
x_{1}^{\prime} \\
x_{2}^{\prime} \\
y^{\prime}
\end{array}\right)=\left(\begin{array}{cccc}
\frac{a}{p} & \frac{a_{1}}{t p} & \frac{a_{2}}{t^{2} p} & \frac{c}{t p} \\
\frac{t(2 a-p)}{2 p} & \frac{a_{1}}{p} & \frac{a_{2}+p}{t p} & \frac{c}{p} \\
\frac{t^{2}(a-p)}{2 p} & \frac{a_{1} t}{2 p} & \frac{a_{2}+2 p}{2 p} & \frac{c t}{2 p} \\
\frac{a^{\prime} t}{p} & \frac{a_{1}^{\prime}}{p} & \frac{a_{2}^{\prime}}{t p} & \frac{c^{\prime}}{p}
\end{array}\right)\left(\begin{array}{c}
x \\
x_{1} \\
x_{2} \\
y
\end{array}\right),
$$

where $t=B_{1}^{(r)} / B^{(r)}$ is a coefficient that depends only on $b$, as can be seen from (3.2). The particular values of the coefficients appearing in the last $4 \times 4$ matrix, for $2 \leq b \leq 7$, are given in Appendix A. For all $b$ considered here, this matrix has only one eigenvalue $\lambda$ larger than 1 , implying that for $r \gg 1$ parameters $x_{r}, x_{1_{r}}, x_{2 r}$, and $y_{r}$ grow as $\lambda^{r}$, and consequently, numbers $A^{(r)}, A_{1}{ }^{(r)}$, $A_{2}{ }^{(r)}$, and $C^{(r)}$ asymptotically behave as $\lambda^{r} B^{(r)}$. 
Numbers of two-leg HWs satisfy recursion relation:

$$
\left(\begin{array}{c}
D^{\prime} \\
D_{1}^{\prime}
\end{array}\right)=\left(\begin{array}{ll}
b_{11} & b_{12} \\
b_{21} & b_{22}
\end{array}\right)\left(\begin{array}{c}
D \\
D_{1}
\end{array}\right)+\left(\begin{array}{c}
F \\
F_{1}
\end{array}\right)
$$

where $b_{i j}$ are polynomials in $B^{(r)}$ and $B_{1}^{(r)}$ of the power $b(b+1) / 2-1$ (see Appendix B), whereas $F$ and $F_{1}$ are of the form: $d_{A A} A^{2}+d_{A A_{1}} A A_{1}+d_{A A_{2}} A A_{2}+$ $d_{A C} A C+d_{A_{1} A_{1}} A_{1}^{2}+d_{A_{1} A_{2}} A_{1} A_{2}+d_{A_{1} C} A_{1} C+d_{A_{2} A_{2}} A_{2}^{2}+$ $d_{A_{2} C} A_{2} C+d_{C C} C^{2}$, with $d_{X Y}$ being polynomials in $B^{(r)}$ and $B_{1}^{(r)}$ of the power $b(b+1) / 2-2$. With new parameters defined as $z_{r}=D^{(r)} / B^{(r)}$ and $z_{1 r}=D_{1}^{(r)} / B^{(r)}$, from (3.5) follows simpler recursion relation:

$$
\left(\begin{array}{c}
z^{\prime} \\
z_{1}^{\prime}
\end{array}\right)=\left(\begin{array}{cc}
m_{11} & m_{12} \\
m_{21} & m_{22}
\end{array}\right)\left(\begin{array}{c}
z \\
z_{1}
\end{array}\right)+\left(\begin{array}{c}
f \\
f_{1}
\end{array}\right)
$$

where $m_{i j}$ are numbers, which do not depend on $r$, but only on $b$, whereas $f$ and $f_{1}$ are polynomials in $x_{r}, x_{1 r}$, $x_{2 r}$ and $y_{r}$. In particular, it can be shown that $m_{11}=$ $m+1, m_{12}=2 n / t, m_{21}=m t / 2, m_{22}=n+1$, with $m=b(b-1) / 2$, and $n=b-1$, whereas $f$ and $f_{1}$ have the form: $a_{x x} x^{2}+a_{x x_{1}} x x_{1}+a_{x x_{2}} x x_{2}+a_{x y} x y+a_{x_{1} x_{1}} x_{1}^{2}+$ $a_{x_{1} x_{2}} x_{1} x_{2}+a_{x_{1} y} x_{1} y+a_{x_{2} x_{2}} x_{2}^{2}+a_{x_{2} y} x_{2} y+a_{y y} y^{2}$, where $a_{X Y}$ are again numbers depending only on the scaling parameter $b$. It turns out that for all $b$ considered here $\lambda^{2}$ is larger than eigenvalues $\lambda_{D}$ of the matrix $m_{i j}$, implying that $z_{r}, z_{1 r} \sim \lambda^{2 r}$.

The number $Z_{O}^{(r+1)}$ of open HWs on the gasket of order $(r+1)$, can be expressed as:

$$
\begin{aligned}
Z_{O}^{(r+1)} & =k_{1} A A_{1} B^{m-3} B_{1}^{n+2}+k_{2} A A_{2} B^{m-2} B_{1}^{n+1}+k_{3} A_{1} A_{2} B^{m-1} B_{1}^{n}+k_{4} A C B^{m-3} B_{1}^{n+2} \\
& +k_{5} A_{1} C B^{m-2} B_{1}^{n+1}+k_{6} A_{2} C B^{m-1} B_{1}^{n}+k_{7} A^{2} B^{m-4} B_{1}^{n+3}+k_{8} A_{1}^{2} B^{m-2} B_{1}^{n+1} \\
& +k_{9} A_{2}^{2} B^{m} B_{1}^{n-1}+k_{10} C^{2} B^{m-2} B_{1}^{n+1}+k_{11} D B^{m-2} B_{1}^{n+2}+k_{12} D_{1} B^{m-1} B_{1}^{n+1}
\end{aligned}
$$

where we have suppressed index $r$ on the right-hand side of this relation, and $k_{i}$ are numbers that depend only on $b$. Substituting established asymptotical behavior of $A_{i}, C$ and $D_{i}$ in the latter expression, one finds that all terms on the right-hand side of equation (3.7) have the same asymptotical form, so that

$$
Z_{O}^{(r+1)} \sim \lambda^{2 r}\left(B^{(r)}\right)^{\frac{b(b+1)}{2}} .
$$

Since in [27] it was shown that numbers $B^{(r)}$ and $B_{1}^{(r)}$ for large $r$ behave as $\omega^{N_{r}}$, where the number of sites $N_{r}$ is given by (3.1), it follows that

$$
Z_{O}^{(r)} \sim \omega^{N_{r}} N_{r}^{\gamma}, \quad \text { with } \quad \gamma=2 \frac{\ln \lambda(b)}{\ln \frac{b(b+1)}{2}} .
$$

The values of $\gamma$ for $2 \leq b \leq 7$ are equal to $2.5237 \ldots, 2.1841 \ldots, 2.3411 \ldots, 2.2461 \ldots, 2.2981 \ldots$, and $2.2755 \ldots$, respectively. One should mention here that number of closed HWs asymptotically behaves as $Z_{C}^{(r)} \sim \omega^{N_{r}}$, as was established in [27], where also a closed formula for $\omega$ was derived.

\section{MODIFIED SIERPINSKI GASKET FRACTALS}

Intrigued by the fact that scaling relations for the numbers of HWs, as well as the corresponding values of the critical exponents, are the same for 3-simplex and SG lattices (Sec. II), here we examine HWs on modified Sierpinski gasket (MSG) fractals. In particular, the only difference between GM fractal and corresponding MSG fractal

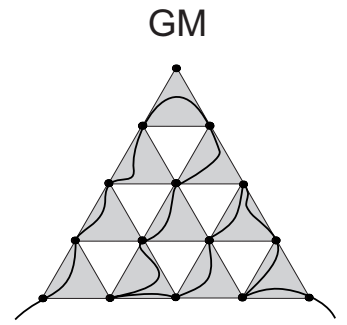

(a)

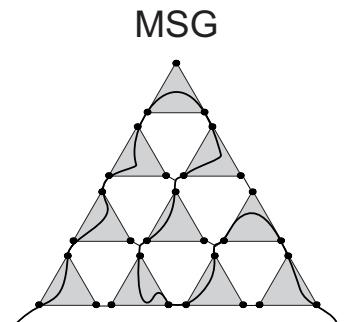

(b)
FIG. 6: (a) The $(r+1)$ th order generator of the GM fractal with $b=4$. Grey triangles represent its 10 constitutive elements, each of them being the $r$ th stage of construction of the fractal ( $r$ th order generator). Most of the corner vertices (black circles) of the $r$ th order generators belong to more than one triangle. (b) The $(r+1)$ th order generator of the MSG fractal with $b=4$ is obtained by connecting $r$ th order generators (grey triangles) via infinitesimal junctions. Curved lines represent two similar $B$-type HWs which in (a) case consists of $B$ and $B_{1}$ type strands within the $r$ th order triangles, whereas in (b) case each strand within the $r$ th order triangle is of $B$ type, implying that all vertices within the triangle are visited (including the third corner vertex).

(with the same value of scaling parameter $b$ ) is that the generator of the MSG fractal is obtained by arranging $b(b+1) / 2$ unit triangles in such a way that vertices of neighboring triangles are connected with an infinitesimal junction, i.e. not glued, as in the GM case (see Fig. 6). This insertion of junctions simplifies recursive scheme for counting HWs: instead of eight different types of walks in the case of GM fractals, one should consider only four 
TABLE I: Coefficients $q, a, c, a^{\prime}$, and $c^{\prime}$, appearing in recursion relations (4.1), and (4.4), for the numbers of $B, A$, and $C$-type HWs, found by direct computer enumeration of all possible corresponding configurations, together with the values of connectivity constant $\omega$ and exponent $\gamma$ for open HWs on MSG fractals with $2 \leq b \leq 8$.

\begin{tabular}{cccccccl}
\hline \hline$b$ & $q$ & $a$ & $c$ & $a^{\prime}$ & $c^{\prime}$ & $\omega$ & $\gamma$ \\
\hline 2 & 1 & 2 & 2 & 1 & 3 & 1 & 2.52372 \\
3 & 2 & 6 & 10 & 4 & 8 & 1.04729 & 2.12343 \\
4 & 4 & 24 & 42 & 20 & 40 & 1.05269 & 2.3816 \\
5 & 16 & 134 & 228 & 116 & 196 & 1.06824 & 2.23644 \\
6 & 68 & 932 & 1460 & 886 & 1408 & 1.07286 & 2.32218 \\
7 & 464 & 8656 & 12524 & 8372 & 11604 & 1.07875 & 2.27302 \\
8 & 3838 & 101612 & 133764 & 103258 & 133428 & 1.08177 & 2.29907 \\
\hline \hline
\end{tabular}

types of walks in this case.

Any closed HW within the $(r+1)$ th order generator of MSG can be decomposed into $b(b+1) / 2$ strands, each of them being a $B$-type HW within one of the $b(b+1) / 2 r$ th order triangle inside it. Here, $B$-type $\mathrm{HW}$ has the same meaning as in the case of 3 -simplex lattice, i.e. it is a HW that enters a generator at one of its corner vertices, and leaves it through another one, meanwhile visiting all the remaining vertices, including the third corner vertex (see Fig. 6). Recursion relation for the numbers of $B$-type walks is of the form

$$
B^{(r+1)}=q\left(B^{(r)}\right)^{\frac{b(b+1)}{2}},
$$

where $q$ depends only on $b$, and, consequently, for $r>>1$, the number $B^{(r)}$ behaves as:

$$
B^{(r)} \sim\left[q^{\frac{2}{(b-1)(b+2)}}\right]^{\left(\frac{b(b+1)}{2}\right)^{r}} .
$$

The number of closed HWs on the $(r+1)$ th order generator $Z_{C}^{(r+1)}$ is equal to:

$$
Z_{C}^{(r+1)}=\operatorname{const}\left(B^{(r)}\right)^{\frac{b(b+1)}{2}}
$$

meaning that $Z_{C}^{(r)}$ has the same asymptotic form as $B^{(r)}$. Since the number $N_{r}$ of sites on the $r$ th order generator is equal to $N_{r}=3[b(b+1) / 2]^{r}$, for large $r$ one gets the following scaling form:

$$
Z_{C}^{(r)} \sim B^{(r)} \sim \omega^{N_{r}}, \quad \text { with } \omega=q^{\frac{2}{3(b-1)(b+2)}} .
$$

Values of $q$ and $\omega$ for $2 \leq b \leq 8$ are given in Table $\Pi$

The number $Z_{O}^{(r+1)}$ of open HWs on the $(r+1)$ th order generator is equal to:

$$
Z_{O}^{(r+1)}=B^{\frac{b(b+1)}{2}-2}\left(k_{1} A^{2}+k_{2} A C+k_{3} C^{2}+k_{4} B D\right),
$$

where $A=A^{(r)}, B=B^{(r)}, C=C^{(r)}$ and $D=D^{(r)}$ have the same meaning as in the case of 3 -simplex lattice, and $k_{i}$ are some constants depending only on $b$. Numbers $A$ and $C$ for two consecutive stages of MSG fractal construction satisfy the recursion relation:

$$
\left(\begin{array}{l}
A^{\prime} \\
C^{\prime}
\end{array}\right)=\left(B^{(r)}\right)^{\frac{b(b+1)}{2}-1}\left(\begin{array}{cc}
a & c \\
a^{\prime} & c^{\prime}
\end{array}\right)\left(\begin{array}{l}
A \\
C
\end{array}\right),
$$

where $a, c, a^{\prime}$, and $c^{\prime}$ depend only on $b$, and can be found by enumeration of the corresponding one-leg HWs (see Table . Dividing this relation by recursion relation (4.1) for the $B$ numbers, and introducing new variables: $x_{r}=$ $A^{(r)} / B^{(r)}$, and $y_{r}=C^{(r)} / B^{(r)}$, one gets

$$
\left(\begin{array}{l}
x_{r+1} \\
y_{r+1}
\end{array}\right)=\frac{1}{q}\left(\begin{array}{cc}
a & c \\
a^{\prime} & c^{\prime}
\end{array}\right)\left(\begin{array}{l}
x_{r} \\
y_{r}
\end{array}\right)
$$

implying that for $r \gg 1$ parameters $x_{r}$ and $y_{r}$ behave as $\lambda^{r}$, where

$$
\lambda=\frac{1}{2 q}\left(a+c^{\prime}+\sqrt{\left(a-c^{\prime}\right)^{2}+4 c a^{\prime}}\right)
$$

is the larger eigenvalue of the $2 \times 2$ matrix in (4.5).

The number $D^{(r)}$ of two-leg HWs transforms as:

$D^{\prime}=B^{\frac{b(b+1)}{2}-2}\left(d_{A A} A^{2}+d_{A C} A C+d_{C C} C^{2}+d_{D} B D\right)$,

which, with $z_{r}=D^{(r)} / B^{(r)}$ can be rewritten as

$$
z_{r+1}=\frac{1}{q}\left(d_{A A} x_{r}^{2}+d_{A C} x_{r} y_{r}+d_{C C} y_{r}^{2}\right)+\frac{d_{D}}{q} z_{r} .
$$

In a similar way as it was done for two-leg walks in the case of GM fractals (Appendix B) it can be deduced that $d_{D} / q=b(b+1) / 2$. Then, equation (4.7) implies that the large $r$ behavior of $z_{r}$ is governed by the larger of numbers $\lambda^{2}$ and $b(b+1) / 2$. By computer enumeration of all possible one-leg configurations we found that inequality $\lambda^{2}>b(b+1) / 2$ is satisfied for $2 \leq b \leq 8$, so that $z_{r} \sim \lambda^{2 r}$.

With the established large $r$ behavior of $x_{r}, y_{r}$ and $z_{r}$, and consequent behavior of $A^{(r)}, C^{(r)}$ and $D^{(r)}$, together with the previously found relation (4.3) for $B^{(r)}$, it is not difficult to find out that scaling form for overall number $Z_{O}^{(r)}$ on MSG fractal is the same as in the case of corresponding GM fractal (3.8), but with different values of $\omega$ and $\lambda$. Consequently, the only exception - equality of the values of $\gamma$ for 3 -simplex and $\mathrm{SG}$ fractals $(b=2$ case of MSG and GM fractals, respectively), seems to be accidental. Indeed, this exception is also an indication that number of contacts per monomer is not the only relevant physical measure of HWs frustration.

The particular values of the connectivity constant $\omega$ and the exponent $\gamma$ for MSG fractals with $2 \leq b \leq 8$ are given in Table \ whereas in Fig. [7 we depict $\gamma$ as a function of $1 / b$, for both GM and MSG fractals. One can see that $\gamma$ is not a monotonic function of $b$, which has not been found for any of the critical exponents connected with SAWs on these fractal families [10, 14, 15]. For 


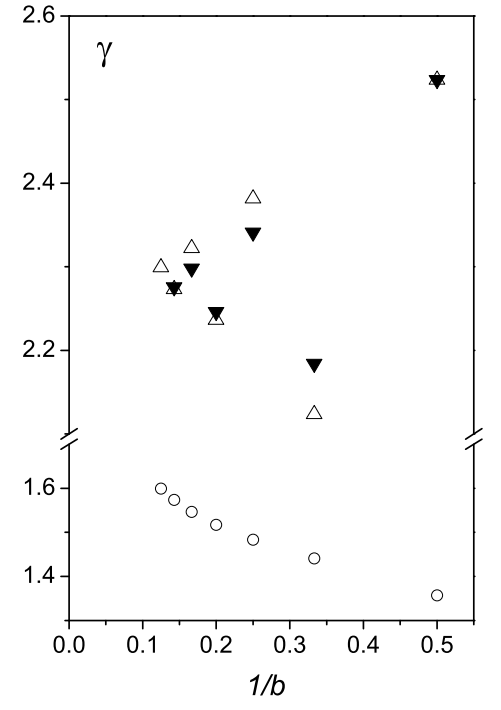

FIG. 7: Values of the exponent $\gamma$ appearing in the scaling form $Z_{O} \sim \omega^{N} N^{\gamma}$ found for the number of open HWs on GM (full triangles) and MSG $(\triangle$ ) fractals, together with the corresponding values for SAWs (o) on the same fractal families [10], as functions of the reciprocal of the fractal parameter $b$.

the sake of comparison on the same figure we give the corresponding values of $\gamma$ for open SAWs [10], which are the same for GM and MSG fractals with equal $b$. In addition, one can also observe that values of $\gamma$ for HWs are separated in two groups: for even values of $b$ exponent $\gamma$ decreases when $b$ grows, whereas for $b$ odd $\gamma$ increases with $b$. This, however, resembles the behavior of the exponent $\psi$, appearing in the scaling form $\lambda^{N} \mu^{N^{\psi}}$ for the average number of different configurations of a branched polymer of $N$ bonds on GM fractal with $b>2$. Namely, for even and odd values of $b$ in a recent paper [32] Dhar obtained two different exact expressions for $\psi$. Finally, it is clearly seen in Fig. 7 that the difference between $\gamma$ values for GM and MSG fractals becomes smaller with larger $b$, so it seems plausible to investigate HWs only on MSG fractals (which is simpler than GM case), in order to obtain the large $b$ behavior of the exponent $\gamma$. This limit is interesting because for large $b$ already at the first stage of the construction of the GM fractal, one gets finite, but large, homogeneous triangular lattice, for which asymptotic form of the number of HWs has not been established yet.

\section{N-SIMPLEX LATTICES}

In order to extend our analysis to higher dimensional lattices, in this section we turn to $n$-simplex fractals with $n>3$, which are embedded in $d=n-1$ dimensional Euclidean spaces. To obtain an $n$-simplex lattice [9] one starts with a complete graph of $n$ points and replaces

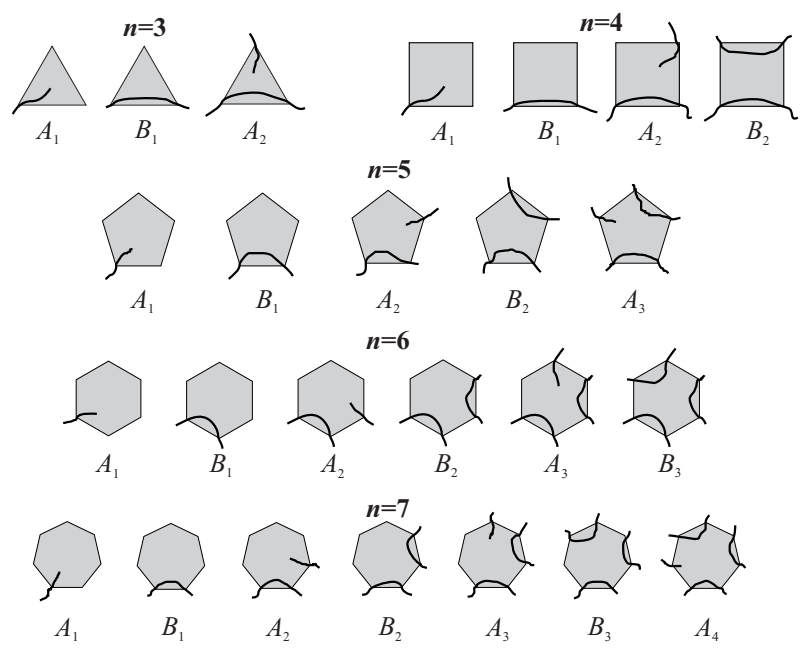

FIG. 8: All types of HWs needed for determining the connectivity constants and asymptotic forms of the numbers of HWs for $n$-simplex lattices with $3 \leq n \leq 7$. Gray polygons denote $n$-simplex of order $l$.

each of these points by a new complete graph of $n$ points. The subsequent stages are constructed self-similarly, by repeating this procedure. After $l$ such iterations one obtains an $n$-simplex of order $l$, which consists of $N_{l}=n^{l}$ points. The complete $n$-simplex lattice is obtained in the limit $l \rightarrow \infty$. Fractal dimension $d_{f}$ of $n$-simplex lattice is equal to $d_{f}=\ln n / \ln 2$.

\section{Closed HWs}

Any closed HW on $n$-simplex of order $l+1$ can be decomposed into $n$ parts within its $n$ simplices of order $l$. Parts (steps) of the walk within the simplices of order $l$ can be of $[n / 2]$ different types (see Fig. 8 ), and we shall denote the numbers of corresponding HWs by $B_{1}, B_{2}$, $\ldots, B_{[n / 2]}$. The overall number of closed HWs is equal to

$$
Z_{C}^{(l+1)}=\sum_{i_{1}+\cdots+i_{\left[\frac{n-1}{2}\right]}=n} c_{i_{1} i_{2} \ldots i_{\left[\frac{n-1}{2}\right]}} \prod_{j=1}^{\left[\frac{n-1}{2}\right]} B_{j}^{i_{j}},
$$

where $c_{i_{1}} i_{2} \cdots i_{[(n-1) / 2]}$ is the number of closed HW configurations with $i_{1}, \ldots, i_{[(n-1) / 2]}$ steps of $B_{1}, \ldots, B_{[(n-1) / 2]}$ type, respectively. Numbers $B_{i}$ satisfy a closed set of recursion relations:

$$
\begin{aligned}
B_{i}^{(l+1)} & =\sum_{i_{1}+\cdots+i_{[n / 2]}=n} b_{i_{1} i_{2} \cdots i_{[n / 2]}}^{(i)} \prod_{j=1}^{[n / 2]} B_{j}^{i_{j}}, \\
i & =1,2, \cdots,[n / 2]
\end{aligned}
$$

where $b_{i_{1} i_{2} \cdots i_{[n / 2]}}^{(i)}$ is the number of HW configurations of $B_{i}$-type that traverse the simplex of order $(l+1)$, and have $i_{1}, \ldots, i_{[n / 2]}$ steps of $B_{1}, \ldots, B_{[n / 2]}$ type, respectively. 
Due to the self-similarity of the underlying lattice both $c_{i_{1} i_{2} \cdots i_{[(n-1) / 2]}}$ and $b_{i_{1} i_{2} \cdots i_{[n / 2]}}^{(i)}$ depend only on $n$. Starting with the initial values $B_{1}^{(0)}=1, B_{2}^{(0)}=\cdots=B_{[n / 2]}^{(0)}=$ 0 , one can iterate recursion relations (5.2) and calculate $B_{i}^{(l)}$ and $Z_{C}^{(l)}$, in principle for any $l$, but since all these numbers grow very fast with $l$, it is useful to introduce new parameters: $x_{i}=B_{i} / B_{[n / 2]}, i=1, \ldots,[n / 2]-1$. Dividing relations for $B_{i}^{(l+1)}$ by $B_{[n / 2]}^{(l+1)}$ one obtains new recursion relations:

$$
x_{i}^{(l+1)}=\frac{f_{i}\left(x_{1}^{(l)}, \ldots, x_{[n / 2]-1}^{(l)}\right)}{f\left(x_{1}^{(l)}, \ldots x_{[n / 2]-1}^{(l)}\right)},
$$

where

$$
\begin{aligned}
& f_{i}\left(x_{1}^{(l)}, \ldots, x_{[n / 2]-1}^{(l)}\right)=B_{i}^{(l+1)} /\left(B_{[n / 2]}^{(l)}\right)^{n}, \\
& f\left(x_{1}^{(l)}, \ldots x_{[n / 2]-1}^{(l)}\right)=B_{[n / 2]}^{(l+1)} /\left(B_{[n / 2]}^{(l)}\right)^{n}
\end{aligned}
$$

$i=1, \ldots,[n / 2]-1$. Iterating these relations (starting with $l=1$ ) one notices that large $l$ behavior of $x_{i}^{(l)}$ strongly depends on the parity of $n$. It turns out that for odd $n$ values all $x_{i}^{(l)}$ tend to some finite constants, whereas for even $n$ the following asymptotic relations are valid:

$$
x_{i}^{(l)} \sim\left(\lambda_{B}{ }^{k-i}\right)^{2^{l}}, \quad i=1, \cdots, k-1,
$$

where $k=[n / 2]$ and $\lambda_{B}$ is some finite constant less than 1. Such behavior is a direct consequence of the fact that for even values of $n$ none of $B_{1}, B_{2}, \ldots, B_{k-1}$-type configurations within a simplex of order $(l+1)$ can be accomplished by $n$ steps of $B_{k}$-type through comprising $n$ simplices of order $l$ (see Appendix [C), which is not the case for simplices with odd $n$, because $B_{i}$-type steps occupy even number of corner vertices of any simplex.

With the established large $l$ behavior of numbers $x_{i}$ it can be shown that from (5.1) follows

$$
Z_{C}^{(l+1)} \sim \begin{cases}\left(B_{k}^{(l)}\right)^{n}, & \text { for } n \text { odd } \\ \left(\lambda_{B}^{2^{l}} B_{k}^{(l)}\right)^{n}, & \text { for } n \text { even }\end{cases}
$$

Although these two asymptotic forms are not the same, from the definition (1.2) of the connectivity constant $\omega$ in both cases one gets

$$
\ln \omega=\lim _{l \rightarrow \infty} \frac{\ln Z_{C}^{(l+1)}}{n^{l+1}}=\lim _{l \rightarrow \infty} \frac{\ln B_{[n / 2]}^{(l)}}{n^{l}} .
$$

The last limiting value can be obtained via numerical iteration of the recursion relation

$$
\frac{\ln B_{k}^{(l+1)}}{n^{l+1}}=\frac{\ln B_{k}^{(l)}}{n^{l}}+\frac{1}{n^{l+1}} \ln f\left(x_{1}^{(l)}, \ldots x_{k-1}^{(l)}\right),
$$

which follows directly from (5.4). Furthermore, from (5.7) one has

$$
\frac{\ln B_{k}^{(p)}}{n^{p}}=\frac{\ln B_{k}^{(l)}}{n^{l}}+\sum_{m=l}^{p-1} \frac{1}{n^{m+1}} \ln f\left(x_{1}^{(m)}, \ldots, x_{k-1}^{(m)}\right),
$$

which, with $p \rightarrow \infty$, gives

$$
\ln \omega=\frac{\ln B_{k}^{(l)}}{n^{l}}+\sum_{m=l}^{\infty} \frac{1}{n^{m+1}} \ln f\left(x_{1}^{(m)}, \ldots x_{k-1}^{(m)}\right) .
$$

Then, since $f\left(x_{1}^{(m)}, \ldots x_{k-1}^{(m)}\right)$ decreases with $m$, it follows that $n^{l} \ln \omega-$ const $<\ln B_{k}^{(l)}<n^{l} \ln \omega$, i.e. $\ln B_{k}^{(l)} \sim$ $n^{l} \ln \omega$. Using this, together with (5.6), for the numbers of closed HWs one finally obtains the scaling formulas

$$
Z_{C}^{(l)} \sim \begin{cases}\omega^{N_{l}}, & \text { for } n \text { odd, } \\ \omega^{N_{l}}\left(\lambda_{B}^{n / 2}\right)^{N_{l}^{\sigma}}, & \text { for } n \text { even, }\end{cases}
$$

where $\sigma=\frac{\ln 2}{\ln n}=\frac{1}{d_{f}}$, with $d_{f}$ being the fractal dimension of the $n$-simplex lattice. Scaling forms and particular values for $\omega$ were found in [26] for $n=4$, in [27] for $n=5$, and 6 , and for $n=7$ in Appendix Cof this paper.

\section{Open HWs}

Any open HW on $n$-simplex of order $(l+1)$ can be decomposed into $n$ parts within its $n$ simplices of order $l$. The two ends of the HW are either both in the same or in two different $l$ th order $n$-simplices. In the first case the configuration of the corresponding $\mathrm{HW}$ part is of the two-leg type, whereas in the latter case the configurations of both ending parts are of the one-leg type. For each $n$ there are $n-[n / 2]$ different types of one-leg configurations (for $3 \leq n \leq 7$ they are depicted in Fig. 8). The remaining $(n-1)$ or $(n-2)$ parts of the open HW correspond to $B_{i}$-type steps. If we denote the numbers of one- and two-leg walks by $A_{i}^{(l)}$ and $D_{i}^{(l)}$, respectively, then the overall number of open HWs on $n$-simplex of order $(l+1)$ is equal to

$$
Z_{O}^{(l+1)}=\sum_{i, j=1}^{n-\left[\frac{n}{2}\right]} E_{i j} A_{i}^{(l)} A_{j}^{(l)}+\sum_{i=1}^{\left[\frac{n}{2}\right]} F_{i} D_{i}^{(l)}
$$

where

$$
\begin{aligned}
E_{i j} & =\sum_{\sum_{r=1}^{[n / 2]} i_{r}=n-2} e_{\left.i_{1} i_{2} \cdots i_{[n / 2]}\right]}^{(i j)} \prod_{r=1}^{[n / 2]}\left(B_{r}^{(l)}\right)^{i_{r}}, \\
F_{i} & =\sum_{\sum_{r=1}^{[n / 2]} i_{r}=n-1} f_{i_{1} i_{2} \cdots i_{[n / 2]}}^{(i)} \prod_{r=1}^{[n / 2]}\left(B_{r}^{(l)}\right)^{i_{r}},
\end{aligned}
$$


with coefficients $e$ and $f$ being the numbers of corresponding HW configurations. Numbers $A_{i}^{(l)}$ of one-leg walks satisfy recursion relations which can be put in the following matrix form

$$
\left(\begin{array}{c}
A_{1}^{(l+1)} \\
\vdots \\
A_{p}^{(l+1)}
\end{array}\right)=\left(\begin{array}{ccc}
a_{11} & \cdots & a_{1 p} \\
\vdots & \ddots & \vdots \\
a_{p 1} & \cdots & a_{p p}
\end{array}\right)\left(\begin{array}{c}
A_{1}^{(l)} \\
\vdots \\
A_{p}^{(l)}
\end{array}\right),
$$

where $p=n-[n / 2]$, and

$$
a_{i j}=\sum_{\sum_{r=1}^{[n / 2]} i_{r}=n-1} \alpha_{\left.i_{1} i_{2} \cdots i_{[n / 2]}\right]}^{(i j)} \prod_{r=1}^{[n / 2]}\left(B_{r}^{(l)}\right)^{i_{r}},
$$

with $\alpha_{i_{1} i_{2} \cdots i_{[n / 2]}}^{(i j)}$ being constant coefficients. Introducing parameters $y_{i}=A_{i} / B_{[n / 2]}, i=1, \ldots, p$, and dividing relations for $A_{i}^{(l+1)}$ by $B_{[n / 2]}^{(l+1)}$ one gets recursion relations for the new parameters:

$$
\left(\begin{array}{c}
y_{1}^{(l+1)} \\
\vdots \\
y_{p}^{(l+1)}
\end{array}\right)=\left(\begin{array}{ccc}
m_{11} & \cdots & m_{1 p} \\
\vdots & \ddots & \vdots \\
m_{p 1} & \cdots & m_{p p}
\end{array}\right)\left(\begin{array}{c}
y_{1}^{(l)} \\
\vdots \\
y_{p}^{(l)}
\end{array}\right),
$$

where

$$
m_{i j}=\frac{a_{i j}\left(B_{1}, \ldots, B_{[n / 2]}\right)}{f\left(x_{1}, \ldots, x_{k-1}\right)\left(B_{[n / 2]}\right)^{n-1}}=\frac{g_{i j}\left(x_{1}, \ldots, x_{k-1}\right)}{f\left(x_{1}, \ldots, x_{k-1}\right)},
$$

with $g_{i j}$ being polynomials of order $n-1$, whose coefficients depend only on $n$, and $f$ defined in (5.4). Numerically iterating relations (5.3) for $x_{i}$, and substituting them into functions $m_{i j}$, after large number of iterations one obtains constant values, such that

- for odd $n$, matrix $m_{i j}$ has only one eigenvalue $\lambda$ larger than 1, implying that $y_{i}^{(l)} \sim \lambda^{l}$,

- for even values of $n$, only $m_{p p} \rightarrow 1$, whereas all other $m_{i j} \rightarrow 0$, implying that $y_{p}^{(l)}$ tends to finite constant value, whereas all the other $y_{i}^{(l)}$ tend to 0 .

Knowing the behavior of parameters $y_{i}^{(l)}$, one can get the large $l$ behavior of the numbers $A_{i}^{(l)}$, and, consequently, the asymptotic form of the first sum in the right-hand side of (5.9). In addition, it can be shown that numbers of two-leg configurations $D_{i}$ are not necessary for establishing the asymptotical behavior of (5.9) (terms with $D_{i}^{(l)}$ either scale as terms with $A_{i}^{(l)} A_{j}^{(l)}$ or they are much smaller). For $n$-simplex lattices with odd $n$ all terms with $A_{i}^{(l)} A_{j}^{(l)}$ have the same asymptotical behavior, whereas for even $n$ behavior of $Z_{O}^{(l+1)}$ is governed by the term with $\left(A_{p}^{(l)}\right)^{2}$. In both cases the following asymptotical formula is valid:

$$
Z_{O}^{(l+1)} \sim\left(y_{p}^{(l)}\right)^{2}\left(x_{[n / 2]-1}^{(l)}\right)^{n-2}\left(B_{[n / 2]}^{(l)}\right)^{n},
$$

which, with obtained behavior of $y_{p}^{(l)}$ and $x_{[n / 2]-1}^{(l)}$, directly gives:

$$
Z_{O}^{(l)} \sim \begin{cases}\omega^{N_{l}} N_{l}^{\gamma}, \quad \gamma=2 \frac{\ln \lambda}{\ln n}, & \text { for odd } n, \\ \omega^{N_{l}}\left(\lambda_{B}^{\frac{n-2}{2}}\right)^{N_{l}^{\sigma}}, \sigma=\frac{\ln 2}{\ln n}, & \text { for even } n .\end{cases}
$$

Details of the derivation of these formulas can be found in Appendix C. Value of $\gamma=2.5237 \ldots$ for $n=3$ was found in Sec. II, whereas for $n=5$ and 7, exponent $\gamma$ is equal to $2.1668 \ldots$ and $2.1079 \ldots$, respectively.

As in GM and MSG fractal cases, one observes that behavior of the number of long HWs on $n$-simplex lattices is strongly affected by the parity of the fractal parameter. For lattices with odd $n$ scaling forms are the same as for GM and MSG fractals. This scaling form coincides with the scaling forms for average numbers of $N$-bonded SAWs on these lattices: with commonly accepted symbols for critical exponents, average number of closed SAWs scales as $\mu^{N} N^{\alpha-3}$, whereas for the average number of open SAWs formula $\mu^{N} N^{\gamma-1}$ is valid (these are the same formulas as for SAWs on homogeneous lattices). Comparing these formulas with those obtained for HWs (and keeping in mind that formulas for HWs correspond to the overall numbers of HWs), one can say that corresponding value $\alpha=2$, obtained for closed HWs on all these lattices, certainly differs from the values obtained for SAWs [9, 10, 12]. The same holds for exponent $\gamma$, meaning that HWs and SAWs belong to different universality classes (see Fig. 77). For HWs on $n$-simplex lattices with even $n$ we obtained different scaling forms: $\omega^{N}\left(\lambda_{B}^{n / 2}\right)^{N^{\sigma}}$ for closed, and $\omega^{N}\left(\lambda_{B}^{(n-2) / 2)}\right)^{N^{\sigma}}$ for open walks. However, one should note here that, whereas polymers on GM, MSG, and 5-simplex fractals for $T>0$ can exist only in swollen phase, on 4- 22], and 6-simplex [24] lattices, bellow some finite temperature $T_{\theta}>0$ polymers collapse into a more compact phase. Scaling of collapsed polymers has not been analyzed so far, and behavior of HWs might be an indication that on $n$-simplex lattices with even $n$, for $T<T_{\theta}$ polymers do not scale in the same manner as they do for $T>T_{\theta}$.

\section{SUMMARY AND CONCLUSION}

In this paper we have analyzed asymptotic behavior of the numbers of open and closed Hamiltonian walks on Given-Mandelbrot, modified Sierpinski gasket and $n$ simplex fractal families. Obtained scaling forms are summarized in Table II. One can see that scaling form (1.1): $Z_{N} \sim \omega^{N} \mu_{S}^{N^{\sigma}} N^{a}$, proposed and obtained for some homogeneous lattices with large number of sites $N$, is satisfied for all fractal lattices under consideration, but, with at least one of the exponents $\sigma$ and $a$ being equal to 0 . For HWs on GM, MSG, and $n$-simplex lattices with odd $n$ scaling form is $\omega^{N}$ for closed, and $\omega^{N} N^{\gamma}(\gamma>0)$ for open walks, i.e. exponent $\sigma$ formally may be taken as 0 . This value cannot be obtained with a simple gen- 
TABLE II: Asymptotic behavior of the numbers of open and closed numbers of HWs on lattices with $N$ sites. Values of $\omega$, $\lambda_{B}$, and $\gamma$ are given in the text, and $d_{f}$ is the fractal dimension of the corresponding lattice.

\begin{tabular}{ccc}
\hline \hline Lattice & Closed HWs & Open HWs \\
\hline \hline $\begin{array}{c}\text { all GM and MSG fractals } \\
n \text {-simplex with odd } n\end{array}$ & $\omega^{N}$ & $\omega^{N} N^{\gamma}$ \\
\hline & \\
$n$-simplex with even $n$ & $\omega^{N}\left(\lambda_{B}^{\frac{n}{2}}\right)^{N^{1 / d_{f}}}$ & $\omega^{N}\left(\lambda_{B}^{\frac{n-2}{2}}\right)^{N^{1 / d_{f}}}$ \\
\hline \hline
\end{tabular}

eralization of the formula $\sigma=(d-1) / d$, valid for $d$ dimensional homogeneous lattice, but it can be explained following the original argument of Owczarek et al [5], which led to (1.1) for collapsed polymers on homogeneous lattices. Namely, collapsed polymer chain on a homogeneous lattice has a form of a compact globule, with a sharp boundary separating it from the surrounding solvent. Monomers on the boundary have smaller number of contacts with other monomers then those in the bulk of the globule, so that a factor $\mu_{S}^{N_{S}}$, with $N_{S}$ being the number of monomers on the boundary, should appear in the scaling form. Furthermore, if one assumes that the boundary itself is homogeneous surface, then $N_{S} \sim N^{(d-1) / d}$, where $N$ is the number of monomers in the polymer chain. The crucial point of this argument is existence of two sets of monomers with different number of contacts. For HWs on both 3-simplex lattice and Sierpinski gasket (MSG and GM with $b=2$, respectively), monomers at all sites, except for the three outer vertices of the lattices, have the same number of contacts (see Figs [1] and 3), so that 'surface' factor with nontrivial $\sigma$ cannot arise. For GM fractals with $b>2$ it can be shown that on the $r$ th stage of fractal construction there are $N_{B}^{(r)}=\frac{b-2}{b}\left\{[b(b+1) / 2]^{r}-1\right\}$ 'bulk' monomers with four contacts, whereas $N_{S}^{(r)}-3=N^{(r)}-N_{B}^{(r)}-3$ 'surface' monomers, with $N^{(r)}$ given by (3.1), have two contacts. This means that for $r \gg 1$ both $N_{B}^{(r)}$ and $N_{S}^{(r)}$ scale as $N^{(r)} \sim[b(b+1) / 2]^{r}$, so that $\mu_{S}^{N_{S}}$ can be incorporated into the term $\omega^{N}$, or formally one can put $\sigma=0$. For HWs on MSG fractals with $b>2$, as well as on $n$-simplex lattices with odd $n$, all sites have the same coordination number (except for the finite number of corner vertices of the whole lattice), and consequently all monomers have the same number of contacts. Hence, the argument of Owczarek et al for these lattices also gives $\sigma=0$. However, presence of the stretched-exponential term $\left(\lambda_{B}^{n / 2}\right)^{N^{1 / d_{f}}}$ for closed, and $\left(\lambda_{B}^{(n-2) / 2)}\right)^{N^{1 / d_{f}}}$ for open Hamiltonian walks on $n$-simplex lattices with even $n$, can't be a consequence of surface effects, since all the sites of these lattices also have the same coordination number. This means that there is some additional effect that should be considered in order to explain obtained scaling forms. It is not clear what that effect is, but one should notice that for $l$ th order $n$-simplex $N_{l}^{1 / d_{f}}=2^{l}$, which is equal to the linear size of the lattice. Also, some clue about it can be achieved by careful inspection of the differences between the asymptotic behavior of the requisite numbers of HWs for lattices with even and odd values of $n$. For instance, for odd $n$ the numbers of long $B_{i}$-type HWs fulfil relation: $B_{1}^{(l)} \sim B_{2}^{(l)} \sim \cdots \sim B_{[n / 2]}^{(l)}$, whereas for even $n$ the number $B_{n / 2}^{(l)}$ is much larger than any other $B_{i}^{(l)}$. Hence, one can expect stretched-exponential terms in scaling forms for HWs on lattices on which 'entangled' configurations are more probable. This bears some analogy with square lattice, for which it was found that large fraction of monomers participate in secondary structures [33.

To conclude, we can say that exact recursive technique, used in this paper for enumeration of all possible Hamiltonian walks on Given-Mandelbrot, modified Sierpinski gasket and $n$-simplex fractal lattices, proved to be very efficient for open, as well as for closed walks. Recursion relations for the number of different types of compact configurations in simpler cases could be obtained by direct enumeration of the corresponding walks, whereas for lattices with more complicated structure we used appropriate computer programs. Once recursion relations were established, it was possible to generate and analyze numbers of very long HWs, eventually obtaining their asymptotical behavior. We believe that obtained scaling forms can help in better understanding of real compact polymers, therefore we intend to apply this technique to HWs on fractals embedded in three-dimensional space, in particular on 3d generalizations of GM and MSG fractals. It would also be interesting to extend the method to interacting HWs. Finally, we hope that our results might be useful in finding scaling forms for collapsed phase of interacting self-avoiding walks on fractals for which transition from swollen to collapsed phase occurs at $T_{\theta}>0$.

\section{Acknowledgments}

We would like to thank I. Živić for careful and critical reading of the manuscript. SEH and SM acknowledge the financial support from the Serbian Ministry of Science and Environmental Protection (Projects No: OI 141020B and OI 144022).

\section{APPENDIX A: RECURSION RELATIONS FOR ONE-LEG HWS ON GM FRACTALS}

In this Appendix we prove that numbers of one-leg HWs: $A, A_{1}, A_{2}$, and $C$, on Given-Mandelbrot fractals, satisfy recursion relations of the form: 


$$
\begin{aligned}
& A^{\prime}=a A B^{m} B_{1}^{n}+a_{1} A_{1} B^{m+1} B_{1}^{n-1}+a_{2} A_{2} B^{m+2} B_{1}^{n-2}+c B^{m+1} B_{1}^{n-1} C, \\
& A_{1}^{\prime}=\left(a-\frac{p}{2}\right) A B^{m-1} B_{1}^{n+1}+a_{1} A_{1} B^{m} B_{1}^{n}+\left(a_{2}+p\right) A_{2} B^{m+1} B_{1}^{n-1}+c B^{m} B_{1}^{n} C, \\
& A_{2}^{\prime}=\frac{a-p}{2} A B^{m-2} B_{1}^{n+2}+\frac{a_{1}}{2} A_{1} B^{m-1} B_{1}^{n+1}+\left(\frac{a_{2}}{2}+p\right) A_{2} B^{m} B_{1}^{n-1}+\frac{c}{2} B^{m-1} B_{1}^{n+1} C,
\end{aligned}
$$

where $p$ is the number of $B$-type (or $B_{1}$-type) configurations within the $(r+1)$ th order generator (see (3.2)), whereas $a, a_{1}, a_{2}$, and $c$ are some integers depending only on the scaling parameter $b$ of the fractal, and $m=b(b-1) / 2, n=b-1$. In order to do that, we first notice that all configurations of $B$-type can be divided in four sets, regarding the last step ("step" is here a part of the walk within the $r$ th-order generator), as is sketched in Fig. 9(a). The number of configurations within each set is denoted by $n_{i}$, so that $n_{1}+n_{2}+n_{3}+n_{4}=p$. Each of the configurations from any of these four sets can be transformed into one $A$-type configuration by cutting the last step. In such a way, if the last step was $B$-type it is converted into an $A$-type step, whereas a $B_{1}$-type step is converted into an $A_{1}$-type step. Number of configurations obtained in this manner, contributing to $A^{(r+1)}$, should be doubled, due to symmetry (there are two corner $r$ th order generators in which $A$-type walk can terminate). This means that $\left(n_{2}+n_{4}\right)\left(B^{(r)}\right)^{m+1}\left(B_{1}^{(r)}\right)^{n}$ HWs of $B$ type on $(r+1)$ th order generator can be transformed into $2\left(n_{2}+n_{4}\right)\left(B^{(r)}\right)^{m}\left(B_{1}^{(r)}\right)^{n} A^{(r)}$ HWs of $A$-type on the same generator. The remaining $\left(n_{1}+n_{3}\right)\left(B^{(r)}\right)^{m+1}\left(B_{1}^{(r)}\right)^{n}$ HWs of $B$-type on the $(r+1)$ th order generator can be converted into $2\left(n_{1}+n_{3}\right)\left(B^{(r)}\right)^{m+1}\left(B_{1}^{(r)}\right)^{n-1} A_{1}^{(r)} \mathrm{HWs}$ of $A$-type (Fig. 9(a)).

In addition, an $A$-type configuration can have its last step in an interior $r$ th order generator, and, as can be seen in Fig. 9(b), that step can be of any of the four possible one-leg types. Number of such walks with the $A$-type end is of the form $n_{A}\left(B^{(r)}\right)^{k}\left(B_{1}^{(r)}\right)^{l} A^{(r)}$, where $k$ and $l$ are numbers of $B$ and $B_{1}$-type steps, respectively. Numbers $k$ and $l$ must satisfy equation $k+l+1=b(b+1) / 2-2$, because every $r$ th order triangle must be traversed by $\mathrm{HW}$, and also $k+2 l+2=(b+1)(b+2) / 2-2$, because every corner vertex of every $r$ th order generator within the $(r+1)$ th order generator, with the exception of two outer vertices, must be visited. The only solution of these two equations is $k=m, l=n$. In a similar way one can obtain the numbers of walks with the $A_{1}, A_{2}$ or $C$-type end in interior $r$ th order generators, so that the number $A^{(r+1)}$ is equal to

$$
\begin{aligned}
A^{(r+1)} & =\left(2 n_{1}+2 n_{3}+n_{A 1}\right) B^{m+1} B_{1}^{n-1} A_{1} \\
& +\left(2 n_{2}+2 n_{4}+n_{A}\right) B^{m} B_{1}^{n} A \\
& +n_{A 2} B^{m+2} B_{1}^{n-2} A_{2}+n_{C} B^{m+1} B_{1}^{n-1} C .
\end{aligned}
$$

On the other hand, from Fig. 9(a) it is quite obvious that $n_{1}=n_{2}$ and $n_{3}=n_{4}$, implying that $n_{1}+n_{3}=n_{2}+n_{4}=$ $p / 2$, and consequently:

$$
\begin{aligned}
A^{(r+1)} & =\left(p+n_{A}\right) B^{m} B_{1}^{n} A+\left(p+n_{A 1}\right) B^{m+1} B_{1}^{n-1} A_{1} \\
& +n_{A 2} B^{m+2} B_{1}^{n-2} A_{2}+n_{C} B^{m+1} B_{1}^{n-1} C .
\end{aligned}
$$

In a similar way, both $A_{1}$ and $A_{2}$-type configurations within the $(r+1)$ th order generator can be divided in two classes: (1) configurations terminating in some interior $r$ th order generator, and (2) configurations terminating in one of the two possible corner $r$ th order generators. The number of $A_{1}$-type configurations in the first class is the same as in the corresponding case of $A$-type configurations, and the only difference between such $A$ and $A_{1}$-type configurations is that $B$ step through one corner triangle should be replaced by $B_{1}$-step. These configurations then give rise to terms $n_{A} B^{m-1} B_{1}^{n+1} A+n_{A 1} B^{m} B_{1}^{n} A_{1}+n_{A 2} B^{m+1} B_{1}^{n-1} A_{2}+$ $n_{C} B^{m} B_{1}^{n} C$ in the relation for $A_{1}^{(r+1)}$. The number of $A_{2}$-type configurations in the first class can be obtained from the corresponding $A$-type configurations by dividing their number by two (because it is predefined in which order the corner vertices are visited) and substituting the two corner $B$-steps by $B_{1}$ steps, thus leading to terms $\left(n_{A} B^{m-2} B_{1}^{n+2} A+n_{A 1} B^{m-1} B_{1}^{n+1} A_{1}+n_{A 2} B^{m} B_{1}^{n-1} A_{2}\right.$ $\left.+n_{C} B^{m-1} B_{1}^{n+1} C\right) / 2$ in the relation for $A_{2}^{(r+1)}$.

As for the second class, lets first consider $A_{1}$-type configurations which visit the upper corner vertex (Fig. 10(a)), and terminate in the right corner triangle. Each of them can be obtained from exactly one $B_{1}$-type configuration, by converting the last $B$ or $B_{1}$-step through the right corner triangle into $A$ or $A_{1}$-step, in a similar way as was done in the case of $A$-type configurations ( $B_{1}$-type configurations can be partitioned in the same way as $B$-type configurations in Fig. 9(a), with the only difference that $B$-step through the upper corner triangle is substituted by $B_{1}$-step). In addition, $A_{1}$-type configurations that visit the upper vertex can terminate in the upper triangle, implying that the last step is of $A_{1}$ or $A_{2}$ type, whereas the step through the right-corner triangle is of type $B$. In Fig. 10(b) it is shown how these configurations can be obtained from $B$-type configurations. Finally, one obtains recursion relation for $A_{1}$-type configurations in the following form:

$$
\begin{aligned}
A_{1}^{\prime} & =\left(\frac{p}{2}+n_{A}\right) B^{m-1} B_{1}^{n+1} A+\left(p+n_{A 1}\right) B^{m} B_{1}^{n} A_{1} \\
& +\left(p+n_{A 2}\right) B^{m+1} B_{1}^{n-1} A_{2}+n_{C} B^{m} B_{1}^{n} C .
\end{aligned}
$$

Since in the case of $A_{2}$-type configurations the order in which corner vertices are visited is fixed, correspond- 


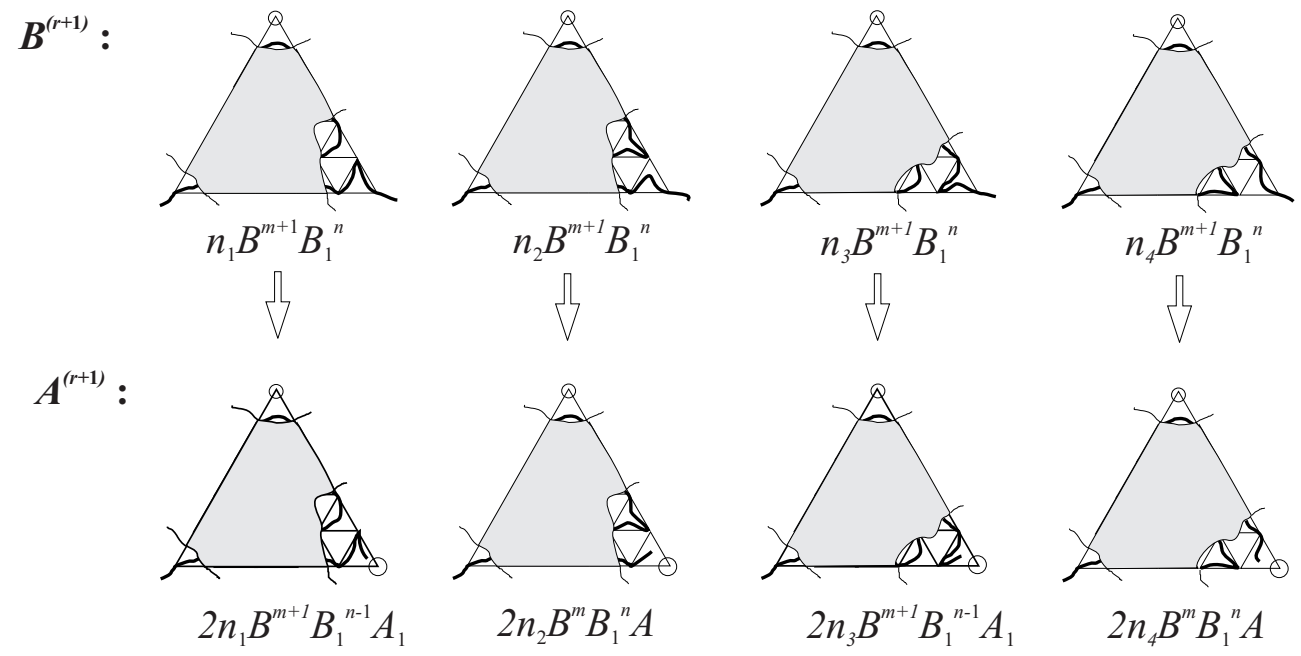

(a)
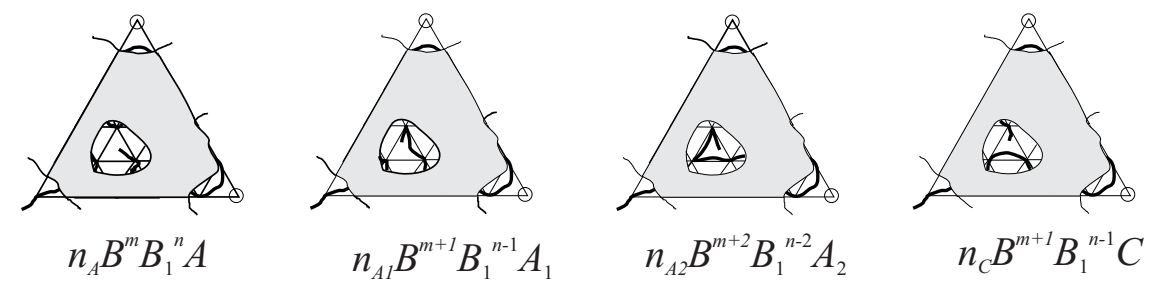

(b)

FIG. 9: (a) Possible configurations of $B$-type HWs on $(r+1)$ th order generator of GM fractal, and corresponding $A$-type HWs, together with the corresponding numbers of walks. Small upward-oriented triangles represent the $r$ th order generators. Encircled nodes are the corner vertices which are not visited by the corresponding HW. (b) Possible types of $A$-type HWs which terminate in interior $r$ th order generators.

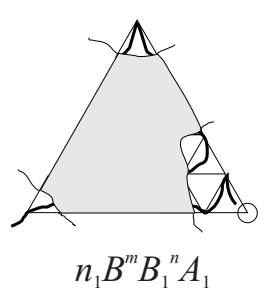

$A_{1}^{(1+1)}$ :

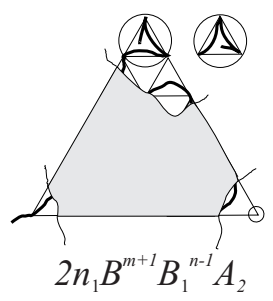

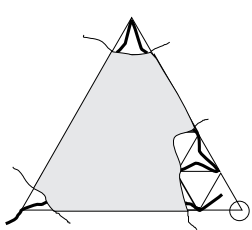

$n_{2} B^{m-1} B_{1}{ }^{n+1} A$

(a)
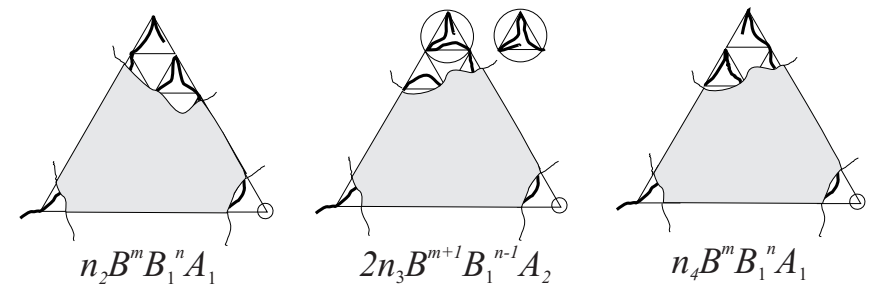

(b)

FIG. 10: Possible configurations of $A_{1}$-type HWs on $(r+1)$ th order generator of GM fractal, which terminate in (a) the right-corner and (b) the upper corner $r$ th order generator, together with the corresponding numbers of walks. 
TABLE III: Coefficients $a, a_{1}, a_{2}, c$, and $p$, appearing in recursion relations (A1) and (3.2) for the numbers of $A, A_{1}$, $A_{2}, B$, and $B_{1}$-type HWs on GM fractals with $2 \leq b \leq 7$, found by computer enumeration of the corresponding $\mathrm{HW}$ configurations.

\begin{tabular}{cccccc}
\hline \hline$b$ & $a$ & $a_{1}$ & $a_{2}$ & $c$ & $p$ \\
\hline 2 & 2 & 2 & 0 & 2 & 2 \\
3 & 12 & 14 & 4 & 22 & 8 \\
4 & 122 & 128 & 36 & 212 & 40 \\
5 & 1842 & 1532 & 436 & 2704 & 360 \\
6 & 35390 & 23812 & 5932 & 42368 & 3872 \\
7 & 880646 & 486284 & 110876 & 878168 & 62848 \\
\hline \hline
\end{tabular}

TABLE IV: Coefficients $a^{\prime}, a_{1}^{\prime}, a_{2}^{\prime}$, and $c^{\prime}$, appearing in recursion relation for the number of $C$-type HWs on GM fractals with $2 \leq b \leq 7$.

\begin{tabular}{ccccc}
\hline \hline$b$ & $a^{\prime}$ & $a_{1}^{\prime}$ & $a_{2}^{\prime}$ & $c^{\prime}$ \\
\hline 2 & 1 & 2 & 2 & 6 \\
3 & 12 & 16 & 8 & 32 \\
4 & 152 & 168 & 96 & 352 \\
5 & 2544 & 2120 & 848 & 4048 \\
6 & 52072 & 35152 & 13136 & 67680 \\
7 & 1340536 & 735312 & 224176 & 1374944 \\
\hline \hline
\end{tabular}

ing walks from the class (2) can terminate only in one of the two corner triangles, lets say in the right corner one. Then, all possible cases, obtained from the partitioning of $B_{1}$-type configurations, are depicted in Fig. 11. These cases, together with configurations that terminate in interior triangles give the relation

$$
\begin{aligned}
A_{2}^{\prime}= & \frac{n_{A}}{2} B^{m-2} B_{1}^{n+2} A+\frac{p+n_{A 1}}{2} B^{m-1} B_{1}^{n+1} A_{1} \\
& +\left(\frac{n_{A 2}}{2}+p\right) B^{m} B_{1}^{n} A_{2}+\frac{n_{C}}{2} B^{m-1} B_{1}^{n+1} C
\end{aligned}
$$

Finally, with $a=p+n_{A}, a_{1}=p+n_{A 1}, a_{2}=n_{A 2}$, and $c=n_{C}$ from (A2), (A3), and (A4), one gets recursion relations $\mathrm{A} 1$. Values of the coefficients $a, a_{1}, a_{2}$, and $c$ for $2 \leq b \leq 7$, are given in Table [II] For $b=2$ and 3 we could find them by direct enumeration of the corresponding HW configurations, whereas for larger $b$ we had to use computer facilities.

In a quite similar manner it can be shown that the number of $C$-type HWs fulfills the recursion relation

$$
C^{\prime}=B^{m} B_{1}^{n}\left(a^{\prime} \frac{B_{1}}{B} A+a_{1}^{\prime} A_{1}+a_{2}^{\prime} \frac{B}{B_{1}} A_{2}+c^{\prime} C\right),
$$

where coefficients $a^{\prime}, a_{1}^{\prime}, a_{2}^{\prime}$, and $c^{\prime}$ depend only on $b$, and their values for $2 \leq b \leq 7$ can be seen in Table IV.

\section{APPENDIX B: RECURSION RELATIONS FOR TWO-LEG HWs ON GM FRACTALS}

Here we prove that the numbers of two-leg HWs, $D$ and $D_{1}$, on GM fractals, satisfy recursion relation (3.5), where coefficients $b_{i j}$ are equal to:

$$
\begin{aligned}
& b_{11}=p(m+1) B^{m} B_{1}^{n}, b_{12}=2 p n B^{m+1} B_{1}^{n-1} \\
& b_{21}=\frac{m p}{2} B^{m-1} B_{1}^{n+1}, b_{22}=p(n+1) B^{m} B_{1}^{n}
\end{aligned}
$$

The two strands which form any two-leg configuration can terminate either in two different $r$ th order generators within the $(r+1)$ th order generator, or in the same one. In the first case, the corresponding number of walks is of the form $B^{k} B_{1}^{l} X Y$, where $X$ and $Y$ are $A, A_{1}, A_{2}$, or $C$, and $k+l+2=b(b+1) / 2$, since all the $r$ th order generators must be visited. In the letter case the number of walks is of the form $B^{k} B_{1}^{l} X$, where $X$ is $D$ or $D_{1}$, and $k+l+1=b(b+1) / 2$. Each such configuration can be obtained by cutting some $B$ or $B_{1}$-step of the $B$ or $B_{1}$ configuration within the $(r+1)$ th generator. All the possibilities in which this can be done are sketched in Fig. 12, so that (B1) directly follows.

\section{APPENDIX C: RECURSION RELATIONS FOR HWS ON $N$-SIMPLEX LATTICES}

In this Appendix we give recursion relations for the requisite numbers of HWs, needed for obtaining the scaling forms for the overall numbers of HWs on $n$-simplex lattices, for $n=4,5,6$, and 7 , together with some relevant details of the derivation of formulas (5.8) and (5.14).

\section{4-simplex}

Closed HWs on 4-simplex were analyzed in [26]. Here we quote equations and results relevant for our present analysis. Recursion relations, as well as the initial values of the numbers $B_{1}^{(l)}$ and $B_{2}^{(l)}$ are

$$
\begin{aligned}
& B_{1}^{\prime}=2 B_{1}^{4}+4 B_{1}^{3} B_{2}+6 B_{1}^{2} B_{2}^{2}, \quad B_{1}^{(1)}=2, \\
& B_{2}^{\prime}=B_{1}^{4}+4 B_{1}^{3} B_{2}+22 B_{2}^{4}, \quad B_{2}^{(1)}=1
\end{aligned}
$$

The requisite number $x \equiv x_{1}=B_{1} / B_{2}$ satisfies recursion relation

$$
x^{\prime}=\frac{B_{1}^{\prime}}{B_{2}^{\prime}}=\frac{2 x^{4}+4 x^{3}+6 x^{2}}{x^{4}+4 x^{3}+22}, \quad x^{(1)}=2 .
$$

Explicit numerical iteration shows that $x^{(l)} \rightarrow 0$, when $l \rightarrow \infty$, so that recursion relation obtains the approximate form

$$
x^{(l+1)} \approx \frac{3}{11}\left(x^{(l)}\right)^{2}
$$




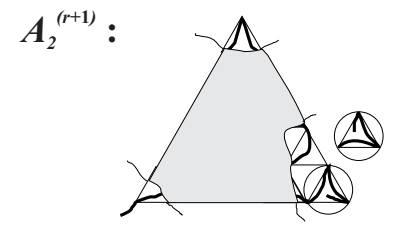

$2 n_{1} B^{m} B_{1}{ }^{n} A_{2}$

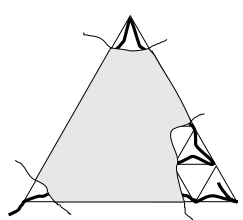

$n_{2} B^{m-1} B_{1}^{n+1} A_{1}$

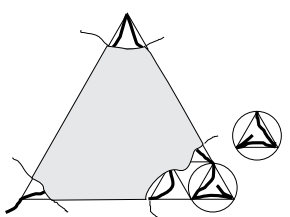

$2 n_{3} B^{m} B_{1}{ }^{n} A_{2}$

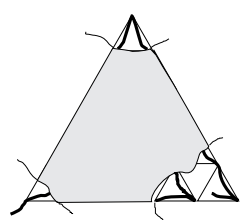

$n_{4} B^{m-1} B_{1}^{n+1} A_{1}$

FIG. 11: Possible configurations of $A_{2}$-type HWs on $(r+1)$ th order generator of GM fractal, with the ending step in the corner $r$ th order generator.
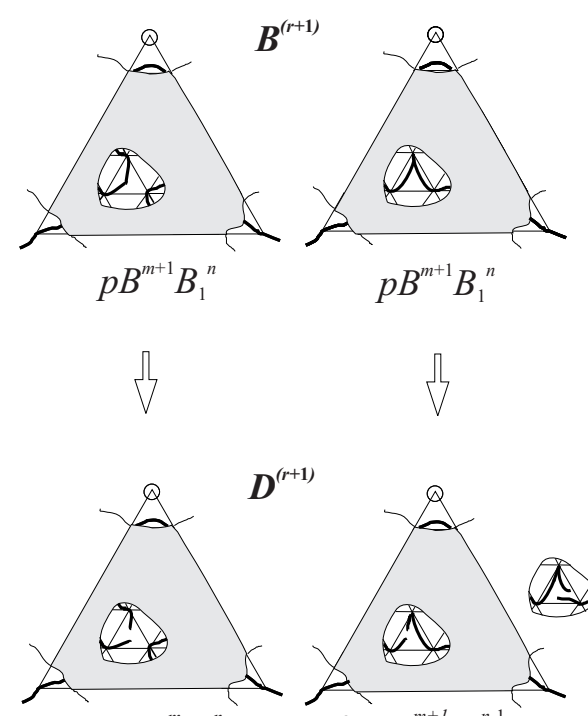

$(m+1) p B^{m} B_{1}{ }^{n} D$

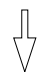

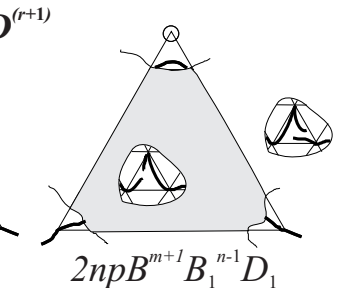

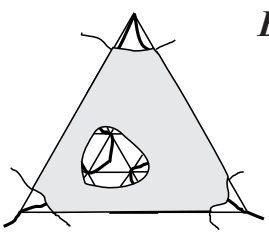

$p B^{m} B_{1}^{n+1}$

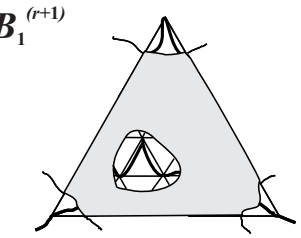

$p B^{m} B_{1}^{n+1}$
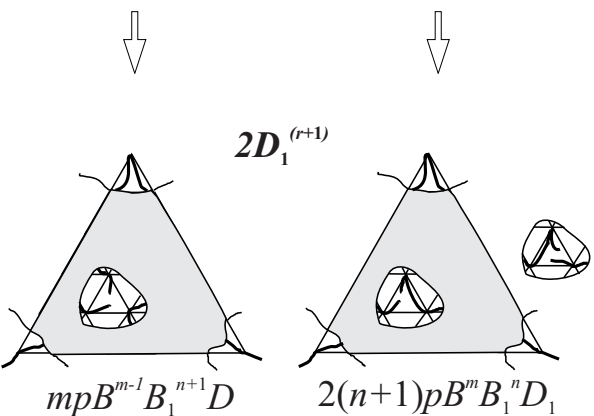

FIG. 12: All possibilities of cutting $B$ or $B_{1}$-type configurations within the $(r+1)$ th order generator of GM fractal, leading to all possible $D$ or $D_{1}$-type configurations, in which the two legs terminate in the same $r$ th order generator. Terms under each configuration are equal to the corresponding walks, within the $(r+1)$ th order generator. One should notice that by cutting steps of $B_{1}$ configurations, due to symmetry, doubled number of $D_{1}$ configurations is obtained (since it is predefined which of the two legs visits the upper corner vertex).

This relation implies that $x^{(l)} \sim \lambda_{B}^{2^{l}}$, and precise numerical analysis gives $\lambda_{B}=0.836620 \ldots$. The overall number of closed HWs on 4-simplex of order $(l+1)$ is equal to

$$
Z_{C}^{(l+1)}=3\left(B_{1}^{(l)}\right)^{4},
$$

which, with established asymptotic behavior of $x^{(l)}$, for large $l$ gives the same form as in (5.6):

$$
Z_{C}^{(l+1)} \sim\left(\lambda_{B}^{2^{l}} B_{2}^{(l)}\right)^{4}
$$

and, consequently, $Z_{C}^{(l)} \sim \omega^{N_{l}}\left(\lambda_{B}^{2}\right)^{N_{l}^{1 / 2}}$, with $\omega=$ $1.39911 \ldots$

Directly enumerating one-leg HW configurations, we have found that numbers $A_{1}$ and $A_{2}$ satisfy recursion relations of the form (5.11) with

$$
\begin{aligned}
& a_{11}=6 B_{1}^{2}\left(B_{1}+B_{2}\right), \quad a_{21}=B_{1}^{2}\left(2 B_{1}+3 B_{2}\right), \\
& a_{22}=22 B_{2}^{2}\left(B_{1}+B_{2}\right)+B_{1}^{2}\left(7 B_{1}+16 B_{2}\right),
\end{aligned}
$$

and $a_{12}=6 a_{21}$, so that for parameters $y_{1}=A_{1} / B_{2}$, and $y_{2}=A_{2} / B_{2}$, one obtains relation (5.13), where

$$
\begin{aligned}
& m_{11}=\frac{6 x^{2}(1+x)}{22+4 x^{3}+x^{4}}, \quad m_{21}=\frac{x^{2}(3+2 x)}{22+4 x^{3}+x^{4}}, \\
& m_{22}=\frac{22+22 x+16 x^{2}+7 x^{3}}{22+4 x^{3}+x^{4}}, \quad m_{12}=6 m_{21} .
\end{aligned}
$$

Since $x^{(l)}=B_{1}^{(l)} / B_{2}^{(l)}$ tends to 0 , it is obvious that $m_{11}$, $m_{12}$, and $m_{21}$ tend to 0 , whereas $m_{22} \rightarrow 1$, when $l \rightarrow$ $\infty$, which implies that $y_{1}$ tends to 0 , and $y_{2}$ to some finite constant value. Explicit simultaneous iteration of relations (C2) and (5.13), starting with the initial values $x^{(1)}=2, y_{1}^{(1)}=6$, and $y_{2}^{(1)}=2$, indeed quickly shows that $y_{1}^{(l)} \rightarrow 0$, and $y_{2}^{(l)} \rightarrow 62.1081 \ldots$.

The overall number of open HWs on $(l+1)$ th order 4 -simplex is equal to

$$
Z_{O}^{(l+1)}=12 B_{1}^{2}\left(A_{1}^{2}+2 A_{1} A_{2}+3 A_{2}^{2}+B_{1} D_{1}\right),
$$

where superscript $(l)$ was suppressed on the right-hand side, and $D_{1}$ is the number of one of two possible two-leg 


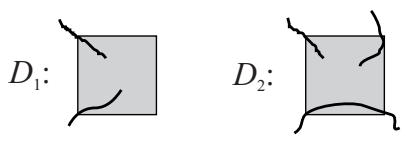

FIG. 13: Possible types $D_{1}$ and $D_{2}$ of two-leg HWs on 4simplex lattice of order $l$.

HWs (see Fig. 13) on the $l$ th order 4 -simplex. To obtain asymptotical behavior of the numbers $D_{1}$ and $D_{2}$, one needs recursion relations:

$$
\left(\begin{array}{c}
D_{1}^{(l+1)} \\
D_{2}^{(l+1)}
\end{array}\right)=\left(\begin{array}{ll}
d_{11} & d_{12} \\
d_{21} & d_{22}
\end{array}\right)\left(\begin{array}{c}
D_{1}^{(l)} \\
D_{2}^{(l)}
\end{array}\right)+\left(\begin{array}{c}
F_{1} \\
F_{2}
\end{array}\right),
$$

where

$$
\begin{aligned}
& d_{11}=12 B_{1} B_{2}^{2}+12 B_{1}^{2} B_{2}+8 B_{1}^{3}, \\
& d_{21}=2 B_{1}^{2}\left(B_{1}+3 B_{2}\right), d_{22}=4 B_{1}^{3}+88 B_{2}^{3},
\end{aligned}
$$

$d_{12}=4 d_{21}$, and $F_{1}=A_{1}^{2} B_{1}\left(4 B_{2}+6 B_{1}\right)+24 A_{1} A_{2} B_{1}\left(B_{2}+\right.$ $\left.B_{1}\right)+A_{2}^{2} B_{1}\left(42 B_{1}+64 B_{2}\right), F_{2}=A_{1}^{2} B_{1}^{2}+6 A_{1} A_{2} B_{1}^{2}+$ $A_{2}^{2}\left(16 B_{1}^{2}+44 B_{1} B_{2}+66 B_{2}^{2}\right)$. Introducing new variables $w_{1}=D_{1} / B_{2}$ and $w_{2}=D_{2} / B_{2}$ one gets new recursion relations, which show that $w_{1}^{(l)} \rightarrow 0$ for $l \rightarrow \infty$. Since from Eq. (C3) follows that

$$
Z_{O}^{(l+1)}=12 x^{2} B_{2}^{4}\left(y_{1}^{2}+2 y_{1} y_{2}+3 y_{2}^{2}+x w_{1}\right),
$$

one finds that two-leg HW configurations do not contribute significantly to the overall number of open HWs for large $l$, and, furthermore, that the following asymptotic formula is valid:

$$
Z_{O}^{(l+1)} \sim\left(x^{(l)}\right)^{2}\left(B_{2}^{(l)}\right)^{4}
$$

Finally, since $B_{2}^{(l)} \sim \omega^{4^{l}}$, and $x^{(l)} \sim \lambda_{B}^{2^{l}}$, one obtains $Z_{O}^{(l)} \sim \omega^{N_{l}} \lambda_{B}^{N_{l}^{1 / 2}}$

\section{5-simplex}

According to [27] recursion relations for requisite numbers $B_{1}$ and $B_{2}$, and their initial values, needed for obtaining the number of closed HWs are such that $x_{1}^{(l+1)} \equiv$ $x^{(l+1)}=B_{1}^{(l+1)} / B_{2}^{(l+1)}=f_{1}\left(x^{(l)}\right) / f\left(x^{(l)}\right), x^{(1)}=3$, with

$$
\begin{aligned}
f_{1} & =6 x^{5}+30 x^{4}+78 x^{3}+96 x^{2}+132 x+132, \\
f & =2 x^{5}+13 x^{4}+32 x^{3}+88 x^{2}+220 x+186 .
\end{aligned}
$$

Numerical iterations give $x^{(l)} \rightarrow 0.8023188 \ldots$, whereas further analysis of relation (5.7) reveals that $\omega=$

\begin{tabular}{|c|c|c|c|c|c|c|c|c|}
\hline \multicolumn{9}{|c|}{ 5-simplex lattice } \\
\hline$i_{1}$ & $i_{2}$ & - & $\alpha_{i_{1} i_{2}}^{(11)}$ & $\alpha_{i_{1} i_{2}}^{(21)}$ & $\alpha_{i_{1} i_{2}}^{(22)}$ & $\alpha_{i_{1} i_{2}}^{(31)}$ & $\alpha_{i_{1} i_{2}}^{(32)}$ & $\alpha_{i_{1} i_{2}}^{(33)}$ \\
\hline 0 & 4 & & 24 & 44 & 548 & 22 & 428 & 1042 \\
\hline 1 & 3 & & 0 & 44 & 636 & 0 & 296 & 472 \\
\hline 2 & 2 & & 72 & 38 & 374 & 0 & 104 & 0 \\
\hline 3 & 1 & & 72 & 24 & 140 & 4 & 36 & 0 \\
\hline 4 & 0 & & 0 & 6 & 26 & 1 & 6 & 5 \\
\hline \multicolumn{9}{|c|}{$\alpha_{i_{1} i_{2}}^{(12)}=12 \alpha_{i_{1} i_{2}}^{(21)}, \alpha_{i_{1} i_{2}}^{(13)}=24 \alpha_{i_{1} i_{2}}^{(31)}, \alpha_{i_{1} i_{2}}^{(23)}=2 \alpha_{i_{1} i_{2}}^{(32)}$} \\
\hline \multicolumn{3}{|c|}{ 6-simplex lattice } & $\alpha_{i_{1} i_{2} i_{3}}^{(11)}$ & $\begin{array}{c}{ }^{6-} \\
\alpha_{i_{1} i_{2} i_{3}}^{(21)} \\
\end{array}$ & $\begin{array}{l}\text { implex } \\
\alpha_{i_{1} i_{2} i_{3}}^{(22)}\end{array}$ & $\begin{array}{l}\text { lattice } \\
\alpha_{i_{1} i_{2} i_{3}}^{(31)}\end{array}$ & $\alpha_{i_{1} i_{2} i_{3}}^{(32)}$ & $\alpha_{i_{1} i_{2} i_{3}}^{(33)}$ \\
\hline 0 & 0 & 5 & 0 & 0 & 0 & 0 & 0 & 541568 \\
\hline 0 & 1 & 4 & 0 & 0 & 0 & 0 & 0 & 541568 \\
\hline 0 & 2 & 3 & 0 & 0 & 57600 & 0 & 47168 & 447232 \\
\hline 0 & 3 & 2 & 0 & 0 & 82848 & 0 & 56768 & 323264 \\
\hline 0 & 4 & 1 & 2640 & 2568 & 66744 & 1042 & 35256 & 148000 \\
\hline 0 & 5 & 0 & 2640 & 1776 & 23688 & 614 & 10220 & 33160 \\
\hline 1 & 2 & 2 & 0 & 0 & 25008 & 0 & 9600 & 47168 \\
\hline 1 & 3 & 1 & 0 & 1776 & 45552 & 472 & 17352 & 58464 \\
\hline 1 & 4 & 0 & 2640 & 2172 & 27768 & 538 & 9576 & 25586 \\
\hline 2 & 1 & 2 & 0 & 0 & 4248 & 0 & 0 & 0 \\
\hline 2 & 2 & 1 & 0 & 624 & 14616 & 0 & 3460 & 6768 \\
\hline 2 & 3 & 0 & 2280 & 1350 & 15360 & 192 & 3976 & 7708 \\
\hline 3 & 0 & 2 & 0 & 0 & 456 & 0 & 0 & 0 \\
\hline 3 & 1 & 1 & 480 & 216 & 2928 & 0 & 480 & 0 \\
\hline 3 & 2 & 0 & 1800 & 612 & 5358 & 68 & 1060 & 1188 \\
\hline 4 & 0 & 1 & 120 & 36 & 300 & 5 & 56 & 0 \\
\hline 4 & 1 & 0 & 720 & 180 & 1182 & 23 & 206 & 168 \\
\hline 5 & 0 & 0 & 120 & 24 & 126 & 3 & 20 & 21 \\
\hline \multicolumn{9}{|c|}{$\alpha_{i_{1} i_{2} i_{3}}^{(12)}=20 \alpha_{i_{1} i_{2} i_{3}}^{(21)}, \alpha_{i_{1} i_{2} i_{3}}^{(13)}=120 \alpha_{i_{1} i_{2} i_{3}}^{(31)}, \alpha_{i_{1} i_{2} i_{3}}^{(23)}=6 \alpha_{i_{1} i_{2} i_{3}}^{(32)}$} \\
\hline
\end{tabular}
$1.717769 \ldots$, and $B_{2}^{(l)} \sim \omega^{5^{l}}$. Since

$$
Z_{C}^{(l+1)}=12 B_{1}^{5}+30 B_{1}^{4} B_{2}+60 B_{1}^{3} B_{2}^{2}+132 B_{2}^{5},
$$

it is obvious that for large $l$ one obtains $Z_{C}^{(l+1)} \sim\left(B_{2}^{(l)}\right)^{5}$, and consequently $Z_{C}^{(l)} \sim \omega^{5^{l}}$.
TABLE V: Coefficients $\alpha^{(i j)}$ given by $(5.12)$, which appear in the recursion relations (5.11), for 5- and 6-simplex lattices.

For 5-simplex lattice there are three types of oneleg configurations, $A_{1}, A_{2}$, and $A_{3}$, which, as we have found by computer enumeration, fulfil recursion relations (5.11), with coefficients $\alpha_{i_{1} i_{2}}^{(i j)}$ (see Eq. (5.12) ) given in Table V. Parameters $y_{i}^{(l)}=A_{i}^{(l)} / B_{2}^{(l)}$ satisfy matrix relation (5.13), with all coefficients $m_{i j}$ tending to finite constant values when $l \rightarrow \infty$, such that corresponding matrix has one eigenvalue larger than one: $\lambda=5.718 \ldots$. This means that $y_{i}^{(l)} \sim \lambda^{l}$, which was also confirmed by direct iteration of the recursion relations for $x$ and $y_{i}$.

Overall number $Z_{O}^{(l+1)}$ of open HWs is of the form (5.9), and, similar to the 3-simplex case, it turns out that terms with the numbers $D_{i}$ of two-leg HWs have the same large $l$ behavior as the terms with numbers of one-leg HWs. Consequently, explicit quoting of recursion relations for $D_{i}$ numbers is not necessary here, and for the number of open HWs one obtains: $Z_{O}^{(l+1)} \sim$ $\left(B_{2}^{(l)}\right)^{3} A_{i}^{(l)} A_{j}^{(l)} \sim \lambda^{2 l}\left(B_{2}^{(l)}\right)^{5}$. Then, straightway follows 
that $Z_{O}^{(l)} \sim \omega^{N_{l}} N_{l}^{\gamma}$, with $\gamma=2 \ln \lambda / \ln 5=2.1668 \ldots$.

\section{6-simplex}

From the recursion relations for the numbers $B_{1}^{(l)}$, $B_{2}^{(l)}$, and $B_{3}^{(l)}$, obtained in [27], certainly follows that $x_{1}^{(l+1)}=f_{1}\left(x_{1}^{(l)}, x_{2}^{(l)}\right) / f\left(x_{1}^{(l)}, x_{2}^{(l)}\right)$, and $x_{2}^{(l+1)}=$ $f_{2}\left(x_{1}^{(l)}, x_{2}^{(l)}\right) / f\left(x_{1}^{(l)}, x_{2}^{(l)}\right)$, with $f_{1}, f_{2}$, and $f$ being polynomials of order 6 . Iterating these relations one concludes that both $x_{1}^{(l)}$, and $x_{2}^{(l)}$ tend to 0 , and also $x_{2}^{(l)} \gg x_{1}^{(l)}$, so that for large $l$ the following approximate relations are valid:

$$
x_{1}^{(l+1)} \approx \frac{25008}{541568}\left(x_{2}^{(l)}\right)^{4}, \quad x_{2}^{(l+1)} \approx \frac{94336}{541568}\left(x_{2}^{(l)}\right)^{2} .
$$

From the latter of these relations follows $x_{2}^{(l)} \sim \lambda_{B}^{2^{l}}$, and then, from the first relation one gets $x_{1}^{(l)} \sim$ $\left(\lambda_{B}^{2}\right)^{2^{l}}$. Precise numerical analysis gives $\lambda_{B}=$ $0.9888 \ldots$ Analysis of the corresponding relation (5.7) gives $\omega=2.0550047 \ldots$, and $B_{3}^{(l)} \sim \omega^{6^{l}}$. Finally, since $Z_{C}^{(l+1)}=60 B_{1}^{6}+360 B_{1}^{5} B_{2}+1170 B_{1}^{4} B_{2}^{2}+1920 B_{1}^{3} B_{2}^{3}+$ $3960 B_{1}^{2} B_{2}^{4}+7920 B_{1} B_{2}^{5}+5580 B_{2}^{6}$, for large $l$ one obtains $Z_{C}^{(l+1)} \approx \operatorname{const}\left(B_{3}^{(l)} x_{2}^{(l)}\right)^{6} \sim\left(\lambda_{B}^{2^{l}} B_{3}^{(l)}\right)^{6}$, and $Z_{C}^{(l)} \sim \omega^{6^{l}}\left(\lambda_{B}^{3}\right)^{\left(6^{l}\right)^{\ln 2 / \ln 6} .}$.

Numbers $A_{1}^{(l)}, A_{2}^{(l)}$, and $A_{3}^{(l)}$ of one-leg HWs for 6simplex lattice satisfy recursion relations (5.11) with coefficients $\alpha_{i_{1} i_{2} i_{3}}^{(i j)}$ given in Table $\mathbf{V}$ Direct numerical iteration of the recursion relations for $x_{i}$ and $y_{i}$ parameters, quickly shows that $y_{1}^{(l)}$ and $y_{2}^{(l)}$ tend to 0 , whereas $y_{3}^{(l)}$ approaches large, but finite constant: $4.08311 \ldots 10^{5}$. This is in accord with the fact that $m_{33}$ is the only element of the matrix $m$ of the relation (5.13) which for large $l$ tends to finite constant, i.e. not to 0 , as do all the other $m_{i j}$. Finally, the overall number (5.9) of open HWs is for large $l$ governed by the term $A_{3}^{2} B_{2}^{4}$, so that $Z_{O}^{(l+1)} \sim\left(x_{2}^{(l)}\right)^{4}\left(B_{3}^{(l)}\right)^{6} \sim\left(\lambda_{B}^{2^{l}}\right)^{4} \omega^{6^{l+1}}$, and $Z_{O}^{(l)} \sim \omega^{6^{l}}\left(\lambda_{B}^{2}\right)^{\left(6^{l}\right)^{\ln 2 / \ln 6} .}$

\section{7-simplex}

To enumerate closed HWs on 7 -simplex lattice one needs three requisite numbers $B_{1}, B_{2}$ and $B_{3}$ (see Fig. 8), which satisfy recursion relations of the form

$$
B_{i}^{(l+1)}=\underbrace{\sum_{i_{1}=0}^{7} \sum_{i_{2}=0}^{7} \sum_{i_{3}=0}^{7}}_{i_{1}+i_{2}+i_{3}=7} b_{i_{1} i_{2} i_{3}}^{(i)} B_{1}^{i_{1}} B_{2}^{i_{2}} B_{3}^{i_{3}}, \quad i=1,2,3 .
$$

Coefficients $b_{i_{1} i_{2} i_{3}}^{(i)}$, found by explicit computer enumeration of the corresponding HW configurations, are given
TABLE VI: Coefficients appearing in recursion relations $B_{i}^{\prime}=$ $\sum b_{i_{1} i_{2} i_{3}}^{(i)} B_{1}^{i_{1}} B_{2}^{i_{2}} B_{3}^{i_{3}}$ for the numbers $B_{i}(i=1,2,3)$, needed

\begin{tabular}{|c|c|c|c|c|c|}
\hline$i_{1}$ & $i_{2}$ & $i_{3}$ & $b_{i_{1} i_{2} i_{3}}^{(1)}$ & $b_{i_{1} i_{2} i_{3}}^{(2)}$ & $b_{i_{1} i_{2} i_{3}}^{(3)}$ \\
\hline 0 & 0 & 7 & 64988160 & 94599168 & 115468800 \\
\hline 0 & 1 & 6 & 64988160 & 141735936 & 165548544 \\
\hline 0 & 2 & 5 & 53667840 & 118798848 & 106301952 \\
\hline 0 & 3 & 4 & 50112000 & 78990336 & 49499520 \\
\hline 0 & 4 & 3 & 32536320 & 38795520 & 19747584 \\
\hline 0 & 5 & 2 & 14097600 & 12925632 & 5819328 \\
\hline 0 & 6 & 1 & 3787680 & 2712192 & 1077136 \\
\hline 0 & 7 & 0 & 473760 & 275616 & 96864 \\
\hline 1 & 0 & 6 & 0 & 6498816 & 11372928 \\
\hline 1 & 1 & 5 & 0 & 10733568 & 9748224 \\
\hline 1 & 2 & 4 & 5660160 & 15033600 & 6708480 \\
\hline 1 & 3 & 3 & 9319680 & 13014528 & 5011200 \\
\hline 1 & 4 & 2 & 7985040 & 7048800 & 2440224 \\
\hline 1 & 5 & 1 & 3459360 & 2272608 & 704880 \\
\hline 1 & 6 & 0 & 626400 & 331632 & 94692 \\
\hline 2 & 1 & 4 & 0 & 566016 & 0 \\
\hline 2 & 2 & 3 & 812160 & 1397952 & 283008 \\
\hline 2 & 3 & 2 & 1953600 & 1597008 & 349488 \\
\hline 2 & 4 & 1 & 1449360 & 864840 & 199626 \\
\hline 2 & 5 & 0 & 396720 & 187920 & 43242 \\
\hline 3 & 1 & 3 & 0 & 54144 & 0 \\
\hline 3 & 2 & 2 & 266880 & 195360 & 20304 \\
\hline 3 & 3 & 1 & 384000 & 193248 & 32560 \\
\hline 3 & 4 & 0 & 163800 & 66120 & 12078 \\
\hline 4 & 1 & 2 & 33600 & 13344 & 0 \\
\hline 4 & 2 & 1 & 80400 & 28800 & 3336 \\
\hline 4 & 3 & 0 & 50400 & 16380 & 2400 \\
\hline 5 & 0 & 2 & 3720 & 672 & 0 \\
\hline 5 & 1 & 1 & 12240 & 3216 & 336 \\
\hline 5 & 2 & 0 & 11520 & 3024 & 402 \\
\hline 6 & 0 & 1 & 840 & 204 & 31 \\
\hline 6 & 1 & 0 & 1680 & 384 & 51 \\
\hline 7 & 0 & 0 & 120 & 24 & 3 \\
\hline
\end{tabular}
for enumeration of closed HWs on 7 -simplex lattice.

in Table [V] whereas initial values are: $B_{1}^{(1)}=120$, $B_{2}^{(1)}=24$, and $B_{3}^{(1)}=3$. Introducing parameters $x_{1}=B_{1} / B_{3}, x_{2}=B_{2} / B_{3}$, one obtains closed set of recursion relations

$$
x_{1}^{\prime}=\frac{f_{1}\left(x_{1}, x_{2}\right)}{f\left(x_{1}, x_{2}\right)}, \quad x_{2}^{\prime}=\frac{f_{2}\left(x_{1}, x_{2}\right)}{f\left(x_{1}, x_{2}\right)},
$$

where

$$
\begin{aligned}
& f_{1}\left(x_{1}, x_{2}\right)=\sum_{i_{1}=0}^{7} \sum_{i_{2}=0}^{7} b_{i_{1} i_{2}\left(7-i_{1}-i_{2}\right)}^{(1)} x_{1}^{i_{1}} x_{2}^{i_{2}}, \\
& f_{2}\left(x_{1}, x_{2}\right)=\sum_{i_{1}=0}^{7} \sum_{i_{2}=0}^{7} b_{i_{1} i_{2}\left(7-i_{1}-i_{2}\right)}^{(2)} x_{1}^{i_{1}} x_{2}^{i_{2}},
\end{aligned}
$$




$$
f\left(x_{1}, x_{2}\right)=\sum_{i_{1}=0}^{7} \sum_{i_{2}=0}^{7} b_{i_{1} i_{2}\left(7-i_{1}-i_{2}\right)}^{(3)} x_{1}^{i_{1}} x_{2}^{i_{2}} .
$$

These recursion relations quickly lead to the conclusion that $x_{1}^{(l)} \rightarrow 0.690015 \ldots$, and $x_{2}^{(l)} \rightarrow 1.14695 \ldots$, as $l \rightarrow \infty$. The limiting values are indeed one of the solutions of the algebraic system of equations $x_{1}=$ $f_{1}\left(x_{1}, x_{2}\right) / f\left(x_{1}, x_{2}\right), x_{2}=f_{2}\left(x_{1}, x_{2}\right) / f\left(x_{1}, x_{2}\right)$, actually the only positive one.

The overall $Z_{C}^{(l+1)}$ number of closed HWs on $(l+1)$ th order 7 -simplex is equal to

$$
Z_{C}^{(l+1)}=\sum_{i_{1}=0}^{7} \sum_{i_{2}=0}^{7} c_{i j(7-i-j)} B_{1}^{i} B_{2}^{j} B_{3}^{7-i-j},
$$

which for large $l$ obtains approximate form:

$$
Z_{C}^{(l+1)} \approx \operatorname{const}\left(B_{3}^{(l)}\right)^{7},
$$

meaning that $\ln Z_{C}^{(l+1)} / 7^{l+1} \sim \ln B_{3}^{(l)} / 7^{l}$. From the recursion relation for $B_{3}^{(l)}$ follows equation:

$$
\frac{\ln B_{3}^{(l+1)}}{7^{l+1}}=\frac{\ln B_{3}^{(l)}}{7^{l}}+\frac{1}{7^{l+1}} \ln f\left(x_{1}^{(l)}, x_{2}^{(l)}\right),
$$

which can be numerically iterated, together with the recursive relations (C5) for $x_{1}$ and $x_{2}$. In that way, one finds $\ln \omega=\lim _{l \rightarrow \infty}\left(\ln B_{3}^{(l)} / 7^{l}\right)=0.87382 \ldots$, i.e. $\omega=2.396056 \ldots$. In addition, from relation (C6) one can write

$$
\frac{\ln B_{3}^{(k)}}{7^{k}}=\frac{\ln B_{3}^{(l)}}{7^{l}}+\sum_{m=l}^{k-1} \frac{1}{7^{m+1}} \ln f\left(x_{1}^{(m)}, x_{2}^{(m)}\right),
$$

so that when $k \rightarrow \infty$ follows

$$
\ln \omega=\frac{\ln B_{3}^{(l)}}{7^{l}}+\sum_{m=l}^{\infty} \frac{1}{7^{m+1}} \ln f\left(x_{1}^{(m)}, x_{2}^{(m)}\right) .
$$

Since $f\left(x_{1}^{(m)}, x_{2}^{(m)}\right)$ decreases with $m$, the above sum is less then $\frac{\ln f\left(x_{1}^{(1)}, x_{2}^{(1)}\right)}{6 * 7^{l}}$, and consequently $7^{l} \ln \omega>$ $\ln B_{3}^{(l)}>7^{l} \ln \omega-5.1$, for all $l$. This means that for $l \gg 1$ one has $\ln B_{3}^{(l)} \sim 7^{l} \ln \omega$, i.e. $B_{3}^{(l)} \sim \omega^{7^{l}}$, finally implying $Z_{C}^{(l)} \sim \omega^{N_{l}}$.

Recursion relations for the numbers $A_{i}^{(l)}$ of four possible one-leg HWs are too cumbersome to be quoted here, but are available upon request. Numerical analysis of corresponding relations (5.13) for parameters $y_{i}^{(l)}=$ $A_{i}^{(l)} / B_{3}^{(l)}$ reveals that $y_{i}^{(l)} \sim \lambda^{l}$, with $\lambda=7.7749 \ldots$, being the largest eigenvalue of the matrix $m_{i j}$, obtained in the limit $l \rightarrow \infty$. All terms in the formula (5.9) for the number $Z_{O}^{(l+1)}$ of open HWs are of the same order of magnitude for large $l$, so that

and, consequently:

$$
Z_{O}^{(l+1)} \sim \lambda^{2 l}\left(B_{3}^{(l)}\right)^{7} \sim \lambda^{2 l} \omega^{7^{l+1}}
$$

$$
Z_{O}^{(l)} \sim \omega^{7^{l}}\left(7^{l}\right)^{\gamma}, \gamma=2 \frac{\ln \lambda}{\ln 7}=2.10791 \ldots
$$

[1] C. Vanderzande, Lattice Models of Polymers (Cambridge University Press, 1998).

[2] J. L. Jacobsen and J. Kondev, Phys. Rev. Lett. 92, 210601 (2004).

[3] R. Lua, A. L. Borovinskiy, and A. Y. Grosberg, Polymer 45, 717 (2004).

[4] B. Duplantier and F. David, J. Stat. Phys. 51, 327 (1988).

[5] A. L. Owczarek, T. Prellberg, and R. Brak, Phys. Rev. Lett. 70, 951 (1993).

[6] A. L. Owczarek, J. Phys. A: Math. Gen. 26, L647 (1993).

[7] M. Baiesi, E.'Orlandini, and A. L. Stella, Phys. Rev. Lett. 96, 040602 (2006).

[8] P. Grassberger and R. Hegger, J. Chem. Phys. 102, 6881 (1995).

[9] D. Dhar, J. Math. Phys. 19, 5 (1978).

[10] S. Elezović, M. Knežević, and S. Milošević, J. Phys. A: Math. Gen. 20, 1215 (1987).

[11] D. Dhar, J. Phys. (Paris) 49, 397 (1988).

[12] S. Kumar, Y. Singh and Y. P. Joshi, J. Phys. A: Math. Gen. 23, 2987 (1990).
[13] S. Kumar and Y. Singh, J. Phys. A: Math. Gen. 23, 5115 (1990).

[14] S. Milošević and I. Živić, J. Phys. A: Math. Gen. 24, L833 (1991).

[15] I. Živić and S. Milošević, J. Phys. A: Math. Gen. 26, 3393 (1993).

[16] F. A. C. C. Chalub, F. D. D. A. Reis, and R. Riera, J. Phys. A: Math. Gen. 30, 4151 (1997).

[17] T. Hatori and T. Tsuda, J. Stat. Phys. 109, 39 (2002).

[18] A. Ordemann, M. Porto, and H. E. Roman, J. Phys. A: Math. Gen. 35, 8029 (2002).

[19] Sumedha and D. Dhar, J. Stat. Phys. 125, 55 (2006).

[20] F. Marini, A. Ordemann, M. Porto, and H. E. Roman, Phys.Rev. E 74, 051102 (2006).

[21] D. J. Klein and W. A. Seitz, J. Physique Lett. 45, L241 (1984).

[22] D. Dhar and J. Vannimenus, J. Phys. A: Math. Gen. 20, 199 (1987).

[23] M. Knežević and J. Vannimenus, J. Phys. A: Math.Gen. 20, L969 (1987).

[24] S. Kumar and Y. Singh, Phys. Rev. A 42, 7151 (1990). 
[25] D. Knežević, M. Knežević, and S. Milošević, Phys. Rev. B 45, 574 (1992).

[26] R. M. Bradley, J. Phys. A: Math. Gen. 22, L19 (1989).

[27] J. Stajić and S. Elezović-Hadžić, J. Phys. A: Math. Gen. 38, 5677 (2005).

[28] R. Rammal, G. Toulouse, and J. Vannimenus, J. Physique 45, 389 (1984).

[29] J. Kondev and J. L. Jacobsen, Phys. Rev. Lett. 81, 2922 (1998).

[30] D. R. Nelson and M. E. Fisher, Ann. Phys. (NY) 91, 226 (1975).
[31] J. A. Given and B. B. Mandelbrot, J. Phys. A: Math. Gen. 16, L565 (1983).

[32] D. Dhar, Phys. Rev. E 71, 031801 (2005).

[33] H. S. Chan and K. A. Dill, Macromolecules 22, 4559 (1989); R. Oberdorf, A. Ferguson, J. L. Jacobsen, and J. Kondev, Phys. Rev. E 74, 051801 (2006).

[34] It can be shown by induction that for general $n$ the following relations are valid: $B_{i}^{(1)}(n)=$ $(n-i-1) ! /(i-1) !, A_{i}^{(1)}(n)=(n-i) ! /(i-1) !$. 